\title{
Review
}

\section{DEMYSTIFYING THE HOLOGRAPHIC MYSTIQUE: A CRITICAL REVIEW}

\author{
D.V. Khveshchenko \\ Department of Physics and Astronomy, University of North Carolina, Chapel Hill, NC 27599, USA \\ E-mail: khvesh@physics.unc.edu
}

Received 26 May 2016; accepted 21 June 2016

\begin{abstract}
Thus far, in spite of many interesting developments, the overall progress towards a systematic study and classification of various 'strange' metallic states of matter has been rather limited. To that end, it was argued that a recent proliferation of the ideas of holographic correspondence originating from string theory might offer a possible way out of the stalemate. However, after almost a decade of intensive studies into the proposed extensions of the holographic conjecture to a variety of condensed matter problems, the validity of this intriguing approach remains largely unknown. This discussion aims at ascertaining its true status and elucidating the conditions under which some of its predictions may indeed be right (albeit, possibly, for a wrong reason).
\end{abstract}

Keywords: strongly correlated systems, holographic correspondence, transport theory, strange metals, analogue gravity PACS: $71.27 .+\mathrm{a}$

\section{Condensed matter holography: the promise}

Among the outstanding grand problems in condensed matter physics is that of a deeper understanding and classification of the so-called 'strange metals' or compressible non-Fermi liquid (NFL) states of the strongly interacting systems. However, despite all the effort and a plethora of the important and nontrivial results obtained with the use of the traditional techniques, this program still remains far from completion.

As an alternate approach, over the past decade there have been numerous attempts inspired by the hypothetical idea of holographic correspondence which originated from string/gravity/high energy theory (where it is known under the acronym AdS/ CFT) to adapt its main concepts to various condensed matter (or, even more generally, quantum manybody) systems at finite densities and temperatures [1-7].

In its original context, the bona fide holographic principle postulates that certain $d+1$-dimensional ('boundary') quantum field theories (e. g. the maximally supersymmetric $\mathrm{SU}(N)$ gauge theory) may allow for a dual description in terms of a string theory which, upon a proper compactification, amounts to a certain $d+2$-dimensional ('bulk') supergravity. Moreover, in the strong coupling limit (characterized in terms of the t'Hooft coupling constant $\lambda=g^{2} N \gg 1$ ) and for a large rank $N \gg 1$ of the gauge symmetry group, the bulk description can be further reduced down to a weakly fluctuating gravity model which can even be treated semiclassically at the lowest (0th) order of the underlying $1 / \mathrm{N}$-expansion.

In the practical applications of the holographic conjecture, the partition function of a strongly interacting boundary theory with the Lagrangian $\mathcal{L}\left(\phi_{\mathrm{a}}\right)$ would then be approximated by a saddle-point (classical) value of the bulk action described by the Lagrangian $L\left(g_{\mu v}, \ldots\right)$ which includes gravity and other fields dual to their boundary counterparts [1-8]

$$
\begin{aligned}
& Z[J]=\int \prod_{a=1}^{N} D \phi_{a} \exp \left(-\int \mathrm{d} t \mathrm{~d}^{d} \vec{x} \mathcal{L}\left(\phi_{a}\right)\right) \approx \\
& \exp \left(-\int \mathrm{d} r \mathrm{~d} t \mathrm{~d}^{d} \vec{x} \mathcal{L}\left(g_{\mu v}, \ldots\right)\right)
\end{aligned}
$$

evaluated with the use of a fixed background metric

$$
\mathrm{d} s^{2}=g_{t t} \mathrm{~d} t^{2}+g_{r r} \mathrm{~d} r^{2}+\sum_{i j} g_{i j} \mathrm{~d} x^{i} \mathrm{~d} x^{j}
$$

while any quantum corrections would usually be neglected by invoking the small parameter $1 / N$. 
Thus, considering that the task of solving a system of coupled Einstein-type differential equations can be fairly straightforward conceptually (albeit not necessarily technically), the holographic approach could indeed become a novel powerful tool for studying the strongly correlated systems and a viable alternative to the practically impossible problem of summing the entire perturbation series. Specifically, if proved valid, some of the broad 'bottom-up' generalizations of the original holographic conjecture known as 'AdS/CMT' (which, in many instances, should have been more appropriately called 'non-AdS/non-CFT') could indeed provide an advanced phenomenological framework for discovering new and classifying the already known types of the NFL behaviour.

Thus far, however, a flurry of the traditionally detailed (hence, rarely concise) publications on the topic have generated not only a good deal of enthusiasm but some reservations as well. Indeed, the proposed ad hoc generalizations of the original string-theoretical construction involve some of its most radical alterations, whereby most of its stringent constraints would have been abandoned in the hope of still capturing some key aspects of the underlying correspondence. This is because the target (condensed matter) systems generically tend to be neither conformally nor Lorentz (or even translationally and/or rotationally) invariant and lack any supersymmetric (or even an ordinary) gauge symmetry with some (let alone, large) rank- $N$ non-abelian group.

Moreover, while sporting a truly impressive level of technical progress, the exploratory 'bottom-up' holographic studies have not yet helped to resolve such crucially important issues as:

- Are the conditions of a large $N$, (super)gauge symmetry, Lorentz/translational/rotational invariance of the boundary (quantum) theory indeed necessary for establishing a holographic correspondence with some weakly coupled (classical) gravity in the bulk?

- Are all the strongly correlated systems (or only a precious few) supposed to have gravity duals?

- What are the gravity duals of the already documented NFLs?

- Given all the differences between the typical condensed matter and string theory problems, what (other than the lack of a better alternative) justifies the adaptation ad verbum of the original (string-theoretical) holographic 'dictionary'? and, most importantly:

- If the broadly defined holographic conjecture is indeed valid, then why is it so?

Considering that by now the field of CMT holography has grown almost a decade old, it would seem that answering such outstanding questions should have been considered more important than continu- ing to apply the formal holographic recipes to an ever increasing number of model geometries and then seeking some resemblance to the real world systems without a good understanding as to why it would have to be there in the first place. In contrast, the overly pragmatic 'shut up and calculate' approach prioritizes computational tractability over physical relevance, thus making it more about the method (which readily provides a plethora of answers but may struggle to specify the pertinent questions) itself, rather than the underlying physics.

On the other hand, there exist, of course, important ongoing efforts towards, both, constructing various 'top-down' holographic models [9-13] as well as trying to derive holography from the already known concepts such as a renormalization procedure on the information-related tensor networks [14-24]. However, the former approach formulated in terms of such objects as D-branes remains to be rather exotic and somewhat hard to connect to from the CMT perspective, whereas the latter one (which, in practice, amounts to a massive use of the Stratonovich and Trotter transformations combined with numerical solutions of the resulting flow equations of the functional RG-type) has yet to deliver a well-defined bulk geometry, other than the basic AdS with the dynamical $z=1$ (or its Lifshitz modification with $z=2$ ), that would be reminiscent of those metrics which are extensively utilized in the 'bottom-up' studies (see below).

\section{Condensed matter holography: the evidence}

The circumstantial evidence that would be typically invoked in support of the general idea of holography includes such diverse topics as thermodynamics of black holes, hydrodynamics of quark-gluon plasma and unitary ultracold Fermi-gases, geometrization of quantum entanglement entropy, etc. However, while possibly attesting to the validity of some aspects of the holographic concept in general, those arguments may not be immediately pertinent to the specific condensed matter systems.

Therefore, for the broadly generalized holographic conjecture to prove relevant and gain a predictive power in the latter context its predictions would have to be systematically contrasted against experimental data as well as the results of other, more conventional, techniques providing a preliminary insight. Also, the holographic calculations would have to be carried out for (and allow for a cross-check between) a whole range of the thermodynamic and transport quantities, including specific heat, DOS, charge and spin susceptibilities, electrical, thermal, and spin conductivities, electron spectral function, etc. 
By some (admittedly, risky) analogy with any evidence claimed to support, e. g. the 'science' of UFO and other paranormal phenomena - most (but not all) of which can be readily dismissed - in order to ascertain the true status of the CMT holography one would need to focus on (and identify the physical origin(s) of) those cases of factual agreement that can be deemed reliable and reproducible. It especially concerns those instances where the holographic predictions were reported to agree quantitatively with the results of some exact analytic [25-29], or almost exact (e. g. Monte Carlo) [30-34] calculations (see below).

At the ad hoc level the holographic approach has already been opportunistically applied to a great variety of condensed matter systems which includes the 'strange' Fermi and Bose metals describing quantum-critical spin liquids, supersolids, quantum smectics and nematics, Mott insulators, (in)coherent conductors, itinerant (anti)ferromagnets, Quantum Hall effect, graphene and other Dirac/Weyl metals, multi-channel Kondo and other quantum impurity models, etc.

Routinely, the holographic calculations would be performed in the 'mean-field' approximation (i. e. at the 0 th order of the would-be $1 / N$-expansion) and then compared to some selected sets of experimental data on the systems that tend to lack any supersymmetry, are characterized by the number of species $N \sim 1$ (such as spin, orbital, and/or valley components) and have only moderate (as opposed to very strong) interactions. The above caveats notwithstanding, however, such studies would often seek nothing short of (and occasionally claim to have found) a quantitative agreement with the data.

Also, many of the early CMT holographic calculations were carried out by 'seeking where the lights are' and utilizing just a handful of the historic black-hole solutions, the central among which is the ReissnerNordstrom (RN) one that asymptotically approaches the $\operatorname{Ad} S_{d+2}$ (anti-de-Sitter) and $\operatorname{Ad} S_{2} \times \mathrm{R}^{d}$ geometries in the UV and IR limits, respectively [1-8],

$$
g_{t t}=-f(r) / r^{2}, g_{r r}=1 / r^{2} f(r), g_{i j}(r)=\delta_{i j} / r^{2},
$$

and is accompanied by the scalar potential $A_{0}=\mu(1-r /$ $\left.r_{\mathrm{h}}\right)$. The emblackening factor $f(r)=1-\left(1+\mu^{2}\right)\left(r / r_{\mathrm{h}}\right)^{d+1}+$ $\mu^{2}\left(r / r_{\mathrm{h}}\right)^{2 d}$ incorporates the chemical potential $\mu$ and vanishes at the horizon of radius $1 / r_{h}$ proportional to the Hawking temperature $T$ which is shared by the bulk and boundary degrees of freedom. Notably, this radius remains finite even when the temperature $T=\left(d+1-(d-1) \mu^{2}\right) / 4 \pi r_{\mathrm{h}}$ vanishes, thereby giving rise to the non-vanishing entropy $S(T \rightarrow 0) \neq 0$ and suggesting that the corresponding boundary theory could provide a description of some isolated 'quan- tum impurity', rather than a correlated many-body state with a non-trivial spatial dispersion.

Accordingly, the boundary fermion propagator $G(\omega, k)$ demonstrates the behaviour dubbed as 'semi-locally critical' [35-44] which is characterized by a non-trivial frequency, yet a rather mundane momentum, dependence

$$
G(\omega, \mathbf{k})=1 /\left(a_{\mathbf{k}}+b_{\mathbf{k}} \omega^{2 \mathbf{v}_{\mathbf{k}}}\right)
$$

where $a_{\mathbf{k}^{\prime}} b_{\mathbf{k}^{\prime}}$, and $v_{\mathbf{k}}$ are smooth functions of the momentum $k$. In the space-time domain, the corresponding behaviour

$$
G(\tau, x) \sim \exp (-S(\tau, x))
$$

is governed by the (semi)classical action $S(\tau, x)=\sqrt{x^{2}+\left(1-2 v_{\mathbf{k}_{\mathrm{F}}}\right)^{2} \ln ^{2} \tau}$ and is consistent with that of the spatially (almost) uncorrelated 'impurities', each of which exhibits a characteristic $d=0$ quantumcritical scaling. Such NFL behaviour indeed bears some superficial resemblance to that found in a certain class of the heavy-fermion compounds [45] and DMFT calculations [46]. However, it is also plagued with such spurious features as potentially multiple Fermi surfaces, dispersionless peaks, and log-oscillating $\omega$-dependence [35-44].

Given the multitude of the experimentally discovered NFLs, the 'locally-critical' scenario would seem to be much too limited to encompass more general types of the real-life NFL scenaria where, both, the $x$ and $\tau$ - (or, correspondingly, $\omega$ - and $k$-) dependences of the propagator would be distinctly non-trivial. In light of that realization, the focus of the early holographic studies has gradually shifted towards a broader class of geometries, including such intrinsically non-Lorentz-invariant metrics as the Lifshitz, Schrödinger, helical Bianchi, etc. A particular attention has been paid to the static, diagonal, and isotropic 'hyperscaling-violating' (HV) metrics with the radial dependence of the form (up to a conformal equivalence),

$$
g_{t t} \sim-r^{2 \theta / d-2 z}, g_{r r}=g_{i i} \sim r^{2 \theta / d-2},
$$

its finite- $T$ version featuring the additional factor $f=1-\left(r / r_{\mathrm{h}}\right)^{d+z-\theta}$. The dynamical exponent $z$ controls the boundary excitation spectrum $\omega \propto q^{z}$, while $\theta$ quantifies a non-trivial scaling of the interval $\mathrm{d} s \rightarrow \lambda^{\theta / d} \mathrm{~d} s$, the scaling-(albeit not Lorentz-) invariant case of the Lifshitz metric corresponding to $\theta=0$. The physically sensible values of $z$ and $\theta$ must satisfy the 'null energy conditions' 


$$
(d-\theta)(d(z-1)-\theta) \geq 0,(z-1)(d+z-\theta) \geq 0
$$

signifying a thermodynamic stability of the corresponding geometry.

A proper choice of $\theta$ determining the effective dimension' $d_{\text {eff }}=d-\theta$ was discussed in the context of fermionic entanglement entropy which singles out the value $\theta=d-1$, consistent with the notion of the Fermi surface as a $d$-1-dimensional membrane in the reciprocal (momentum) space [47-54.

The HV metrics arise among the solutions of various generalized gravity theories, including those with massive vector (Proca) fields, Horava gravity, as well as the Einstein-Maxwell-dilaton (EMD) theory which includes an additional neutral scalar ('dilaton') field [55-61],

$$
\begin{aligned}
& L_{\mathrm{EMD}}=\frac{1}{2}\left(R+\frac{d(d+1)}{L^{2}}\right) \\
& -\frac{(\partial \phi)^{2}}{2}-U(\phi)-\frac{V(\phi)}{2} F_{\mu \nu}^{2}
\end{aligned}
$$

where the dilaton potential $U(\phi)$ and the effective gauge coupling $V(\phi)$ are given by some (typically, exponential) functions of $\phi$. This model is believed to be dual to a strongly interacting boundary theory at finite density and temperature which is deformed away from the hyperscaling limit by a relevant neutral scalar operator dual to the dilaton. The HV solutions of the coupled Einstein-Maxwell equations have also been obtained by taking into account a back-reaction of the fermionic matter fields on the background geometry [62, 63]. It should be noted, though, that apart from a few exceptions [64] such analyses were limited to the hydrodynamic (Thomas-Fermi) description of the fermions, while leaving out their more subtle (exchange and correlation) effects.

One purported success of the theory (8) (with $U(\phi) \sim \phi^{2}$ and $V(\phi)=$ const) was a numerical fit to the experimentally observed power-law behaviour of the mid-infrared optical conductivity [65-68]

$$
\sigma(\omega) \sim \omega^{-2 / 3}
$$

in the normal state of the superconducting cuprates such as BSCYCO [69]. Notably, though, such an agreement was found over less than half of a decade $(2<\omega \tau<8)$ while the later studies did not confirm it [70-72]. Besides, it was also argued to be intermittent with the alternate 'universal', $\sim \omega^{-1}$, behaviour.

Another notable example is the holographic calculation [73, 74] of the numerical prefactor $C$ in the empirical Homes relation between the superfluid density, critical temperature, and normal state conductivity [75]:

$$
\rho_{\mathrm{s}}=C T_{\mathrm{c}} \sigma\left(T_{\mathrm{c}}^{0+}\right) .
$$

This relation is obeyed by a large variety of superconductors, including the weakly coupled (yet, sufficiently disordered) conventional ones where a justification for the use of holography would be rather hard to come by and where the Homes' relation can be shown to hold within the scope of the traditional theory [76]. The holographic theory of Refs. [73, 74] claimed its success with finding the prefactor $C \approx 6.2$ to be close to the experimental values in the cuprates which happen to be $C \approx 8.1$ and 4.4 for the $a b$ - and $c$-axes transport, respectively. Somewhat ironically, though, this would seem like a case where, in the absence of a compelling underlying reason, a perfect quantitative agreement could do more harm than good to the cause.

Arguably, a stronger case could have been made if the range of the possible values of $C$ were unusually narrow, thus potentially hinting at some universality of the result. This, however, does not seem to be the case. Moreover, the holographic calculation of Refs. [73, 74] does not readily reproduce any other empirical relations such as the Schneider's one [77]:

$$
\frac{T_{\mathrm{c}}}{T_{\mathrm{c}}^{*}}=2 \sqrt{\frac{\rho_{\mathrm{s}}}{\rho_{\mathrm{s}}^{*}}}\left(1-\frac{1}{2} \sqrt{\frac{\rho_{\mathrm{s}}}{\rho_{\mathrm{s}}^{*}}}\right) .
$$

Interestingly, the later work claimed a compliance of the holographic results with yet another, Uemura - but not the Homes' - law [78], thereby suggesting that the agreement with the experiment reported in Refs. [73, 74] may have been largely fortuitous.

Also, to further strengthen the case for holography, some works tend to use increasingly more and more complicated models with a larger number of fields while operating under the assumption that the IR physics should be fairly universal and insensitive to such details. The results, on the contrary, indicate that there is little universality, as by varying the contents of the bulk theory one can dramatically alter the IR behaviour - e. g. reproducing on demand the entire phase diagram of the cuprates with all the four main phases as well as the domains of their coexistence in the extended EM model with the additional vector and scalar fields which represent the competing orders [79, 80].

There has also been some effort on the experimental side, such as the report of measuring the shear viscosity-to-entropy density ratio in the cuprates [81] which was found to be close to its celebrated holographic lower bound value [82, 83]: 


$$
\eta / S=1 / 4 \pi
$$

It should be noted, though, that instead of such genuine two-particle characteristic as the correlation function of the stress tensor's component $T_{x y}$ in Ref. [81] the viscosity $\eta$ was deduced from the singleparticle electron spectral function. Furthermore, even the bound (12) itself is known not to be totally universal, its value becoming lower, e. g. in the absence of the spatial rotational invariance [84, 85]:

$$
\eta_{x z x z} / S=(1 / 4 \pi)\left(g_{x x} / g_{z z}\right) .
$$

Yet another example of a successful application of the holographic approach was presented in Refs. [3034. where an impressive agreement was found between the holographically computed frequency-dependent (finite-temperature) conductivity $\sigma(\omega)$ and the Monte Carlo results for the Bose-Hubbard model whose critical behaviour belongs to the universality class of the $\mathrm{O}(2)$-symmetrical Wilson-Fisher critical point. Upon analytically continuing to the real frequencies this agreement gets progressively worse for $\omega<2 \pi T$, though $[86]$. Most intriguingly, the original work of Refs. [30-34] utilized the EM holographic model with a rather special higher-derivative term

$$
\Delta L=C_{a b c d} F^{a b} F^{c d},
$$

where $C_{a b c d}$ is the Weyl tensor, thus making one wonder as to the reasons behind a seemingly unique role of this model as the potential Bose-Hubbard's dual. However, in the later Ref. [86] nearly identical results were obtained with the use of a much less exotic EMD model with $U(\phi)=\phi^{2}$ and $V(\phi)=1+\alpha \phi$. Thus, the previously reported agreement with the MC results (limited to $\omega>2 \pi T$ ) appears to be rather common - an observation which takes away much of the intrigue surrounding the holographic model equipped with the term (14) and makes less pressing the need for understanding its otherwise inexplicably serendipitous success.

\section{Emergent geometry and 'holography light'}

Regardless of those practices in the field of CMT holography that make it prone to criticism, its general idea is undeniably appealing. Indeed, it falls very much in line with some of the most profound paradigms in quantum many-body physics, including those of the bulk-edge correspondence and the notion of running couplings in the process of renormalization alongside the changing scale of energy/length/ information. The former has recently received a lot of attention with the advent of topological insulators/ superconductors and Dirac/Weyl (semi)metals, while the latter provides a standard framework for relating the bare (UV) to the effective (IR) physics.

Arguably, of all the holographic studies the most important is the quest into its possible underlying physical cause(s). This concerns, first and foremost, those situations where some precise - analytic or numerical - agreement has been found between the results of the holographic and some conventional approaches. One such recent example is provided by the random infinite-range fermion-hopping models that were studied in Refs. [25-29], drawing from the previous analyses of the random infinite-range spin-coupling systems [87].

These models also share a number of common features with the Kondo lattice [88, 89] and matrix [90-94] ones, their unifying physical theme being that of a single 'quantum impurity' interacting selfconsistently with a local bath. This behaviour makes them potentially amenable to the description based on the holographic 'semi-locally critical' scenario of Refs. [35-44].

Indeed, in Refs. [25-29] an emergent invariance under the group of reparametrizations was discovered and its relation to the 'semi-local' $\mathrm{AdS}_{2} \times \mathrm{R}^{d}$ geometry was demonstrated by establishing an exact agreement between the holographically computed single-particle propagators in this (non-fluctuating) geometry [35-44] and the two- and four-point correlation functions of certain exactly solvable random fermion-hopping models.

Notably, such a perfect agreement was achieved without invoking a small $1 / N$ parameter, thereby suggesting that the $1 / \mathrm{N}$-corrections (that would have been present in the original string-theoretical AdS/ CFT correspondence) may, in fact, be absent altogether. More precisely, although the large- $N$ limit indeed had to be taken in the random spin-coupling model of Ref. [87] (after taking the limit of a large number $\mathcal{N}$ of the lattice sites), the 'semi-local' behaviour of its random fermion-hopping counterparts sets in already at $\mathcal{N}=1$ (but still for $\mathcal{N} \gg 1$ ) [25-29].

Generalizing the results of Refs. [25-29], one can construct a whole family of 'semi-local' NFL regimes described by the propagator $G(\tau)$ which obeys the integral equations ( $n=2$ in Refs. [25-29])

$$
\begin{aligned}
& \int G\left(\tau_{1}, \tau\right) \Sigma\left(\tau, \tau_{2}\right) \mathrm{d} \tau=\delta\left(\tau_{1}-\tau_{2}\right), \\
& \Sigma\left(\tau_{1}, \tau_{2}\right)=\lambda G^{n}\left(\tau_{1}, \tau_{2}\right) G^{n-1}\left(\tau_{2}, \tau_{1}\right),
\end{aligned}
$$

that are manifestly invariant under an arbitrary change of variables $\tau=f(\sigma)$ : 


$$
G\left(\tau_{1}, \tau_{2}\right)=\left[f^{\prime}\left(\sigma_{1}\right) f^{\prime}\left(\sigma_{2}\right)\right]^{-1 / 2 n} G\left(\sigma_{1}, \sigma_{2}\right) .
$$

To derive these equations from the (disorderaveraged) Hamiltonian of a random infinite-range fermion-hopping model

$$
H=\sum_{k=1}^{n} c_{k} \sum_{a_{i}, b_{i}} \psi_{a_{1}}^{\dagger} \ldots \psi_{a_{k}}^{\dagger} \psi_{b_{1}} \ldots \psi_{b_{k}},
$$

the parameters $c_{k}$ need to be fine-tuned [95], while for their arbitrary values the system conforms to the generic behaviour described by the $n=2$ case. This is somewhat reminiscent of the situation with the exactly soluble $1 d$ spin- $S$ chain model whose behaviour is markedly non-generic for all $S>1 / 2$ [96-98].

Specifically, the solution of Eqs. (15) demonstrates an expressly NFL behaviour

$$
G(\tau) \sim \tau^{-1 / n}
$$

or, equivalently, $\Sigma(\omega) \sim \omega^{1-1 / n}$ which can also be reproduced holographically with the use of a massive bulk fermion subject to the $\mathrm{AdS}_{2} \times \mathrm{R}^{d}$ geometry and described by the action $L_{f}=\mathrm{i} \bar{\psi} \gamma^{\mu} \partial_{\mu} \psi-m \bar{\psi} \psi$ provided that the fermion mass $m$ is chosen as follows:

$$
\Delta_{\psi}=\frac{1}{2}+\left(m^{2} R^{2}-q^{2} \mathcal{E}^{2}\right)^{1 / 2}>1 / 2 n .
$$

Here $R$ is the $\operatorname{Ad} S$ radius, $q$ is the fermion charge, and $\mathcal{E}$ measures the particle-hole asymmetry [25-29]. Thus, regardless of whether or not the 'semi-locally critical' scenario of Refs. [35-44] is immediately applicable to any real-life materials, its predictions might still prove quite useful, as far as the properties of some properly crafted random spin-coupling and fermion-hopping models are concerned.

Notably, though, the dimension (19) arises in the so-called 'unstable' boundary CFT which is dual to $\mathrm{AdS}_{2}$ [35-44], while in the 'stable' one (to which the former is supposed to flow under a double-trace deformation) the operator dimension would be $\Delta_{+}=\frac{1}{2}+\left(m^{2} R^{2}-q^{2} \mathcal{E}^{2}\right)^{1 / 2}>1 / 2$, thus making the value (19) unattainable for any integer $n>1$.

It is also worth noting that the behaviour similar to Eq. (18) can be envisioned in the context of, e. g. sub-Ohmic spin-bath models which, incidentally, can be formulated in terms of the localized Majorana fermions. Among other things, it gives rise to the ubiquitous Lorentzian ('Drude-like') frequency dependences of the various observables, their width being given by the inelastic phase relaxation rate [99-102]. It might be interesting to explore such a possible connection further [95], as well as to reproduce Eq. (18) in the holographic models aspiring to describe the Kondo physics [103-107].
Also, while being characteristic of the purely classical (non-fluctuating) asymptotic near-horizon geometry [35-44] the emergent reparametrization symmetry (15) does not rise to the same level as that in the elaborate constructs of the original (string-theoretical) holographic correspondence where the bulk supports a full-fledged quantum (super)gravity theory that only becomes classical in the large- $N$ limit $[1-6]$. Nevertheless, it is quite remarkable that the quantum systems in question allow for some of their properties to be expressed in purely geometrical terms.

In that regard, emergent classical metrics and concomitant effective gravity-like descriptions are not that uncommon, the most remarkable example being provided by the intriguing relationship between quantum physics and classical geometry in the form of the famous extremal area law of entanglement entropy [108, 109]. To a somewhat lesser extent, same can be said about such connections found in thermodynamics of phase transitions ('Fisher-Ruppeiner metric') 110-113], quantum information and tensor network states [114-120], QHE hydrodynamics ('Fubini-Study metric') [121-123], Chern classes of the Bloch eigenstates of momentum [124-126, Berry phase of the adiabatic time evolution [127-129], etc. In all these different contexts, some underlying invariance under pertinent diffeomorphisms facilitates an elegant and insightful gravitational description.

Importantly, the emergent geometric structures such as effective metrics, curvatures, spin connections, etc. can indeed create the appearance of a limited form of some bulk-boundary correspondence ('holography light') that can be inadvertently mistaken for manifestations of the hypothetical full-fledged CMT holography.

\section{Holographic phenomenology: the cuprates}

Obtaining verifiable holographic predictions can be particularly instructive in those situations where experimental data exhibit robust scaling dependences, thus hinting at a possible (near-)critical regime that might be amenable to a holographic scaling analysis [130]. One such example is provided by the holography-inspired phenomenologies of the cuprates which focused on the robust power-law behaviours of the longitudinal electrical conductivity, Hall angle, and magnetoresistivity that violates the conventional Kohler's law [131]:

$$
\begin{aligned}
& \sigma \sim T^{-1}, \\
& \tan \theta_{\mathrm{H}} \sim T^{-2}, \\
& \Delta \rho / \rho \sim \rho^{2} .
\end{aligned}
$$


In the previously proposed scenaria, Eqs. (20) were argued to indicate a possible existence of two distinctly different scattering times: $\tau_{\mathrm{tr}} \sim 1 / T$ and $\tau_{\mathrm{H}} \sim 1 / T^{2}$ which characterize the longitudinal vs transverse [132] or charge-symmetric vs antisymmetric [133] currents. Yet another insightful proposal was put forward in the framework of the marginal Fermi liquid phenomenology [134].

In contrast to the anomalous transport properties of the cuprates, their thermodynamic ones are more standard, including the Fermi-liquid-like specific heat $C \sim T$ (except for a possible logarithmic enhancement [135]). A recent attempt to rationalize such experimental findings was made in Refs. $136-$ 138]. Under the assumption of an underlying oneparameter scaling the dimensions of the observables were expressed in terms of the minimal set which includes the dynamical critical exponent $z$ alongside the dimensions of the (mass) density $\Delta_{n}$ and effective charge $\Delta_{e}$, the two accounting for, roughly speaking, the wave function renormalization and vertex corrections, respectively.

The linear thermoelectric response is then described in terms of a trio of the fundamental kinetic coefficients

$$
\begin{aligned}
& \mathbf{J}=\hat{\sigma} \mathbf{E}-\hat{\alpha} \nabla T, \\
& \mathbf{Q}=\mathrm{T} \hat{\alpha} \mathbf{E}-\hat{\kappa} \nabla T,
\end{aligned}
$$

where the Onsager's symmetry is taken into account and the off-diagonal entries in the $2 \times 2$ matrices $\hat{\sigma}, \hat{\alpha}, \hat{\kappa}$ represent the Hall components of the corresponding conductivities.

To set up the scaling relations that reproduce Eqs. (20) and other experimentally observed algebraic dependences one has to properly account for the time reversal and particle-hole symmetries. Also, one should be alerted to the fact that the exponents governing the temperature dependences in the kinetic coefficients would be the same as those appearing in their frequency-dependent optical counterparts [130]. However, the previous analyses based on the semiclassical kinetic equation show that such leading (minimal) powers of $T$ may or may not actually survive, depending on whether or not the quasiparticle dispersion and Fermi surface topology conspire to yield comparable rates of the normal and umklapp inelastic scattering processes [139-141]. It would appear, though, that in the cuprates, both, the multi-pocketed (in the underand optimally doped cases) as well as the extended concave (in the overdoped case) hole Fermi surfaces comply with such necessary conditions.
As an additional consistency check, the kinetic coefficients are expected to be consistent with the Fermi liquid relations $S \sim(T / e \sigma) \mathrm{d} \sigma / \mathrm{d} \mu, v_{N} \sim(T / e B) \mathrm{d} \theta_{H} / \mathrm{d} \mu$, where the r.h.s. are proportional to the Fermi surface curvature but do not contain such single-particle characteristics as scattering time or effective mass and, therefore, might be applicable beyond the coherent quasiparticle regime.

It was found in Ref. [138] that, somewhat surprisingly, most of the experimental data favour the rather mundane solution

$$
z=1, \Delta_{e}=0, \Delta_{n}=1
$$

which imposes the following relation on the Seebeck $(S)$, Hall Lorenz $\left(L_{\mathrm{H}}\right)$, and Nernst $\left(v_{\mathrm{N}}\right)$ coefficients (barring any Sondheimer-type cancellations):

$$
[S]+\left[v_{\mathrm{N}}\right]-\left[L_{\mathrm{H}}\right]=0 .
$$

In the optimally doped cuprates, the experimentally measured thermopower, apart from a finite offset term, demonstrates a (negative) linear $T$-dependence [142]. As regards the Hall Lorenz and Nernst coefficients, the data on the untwinned samples of the optimally doped $\mathrm{YBaCuO}$ were fitted into a linear dependence, $L_{\mathrm{H}} \sim T$, whereas $v_{\mathrm{N}}$ was generally found to decrease with increasing $T$, thus suggesting $\left[v_{\mathrm{N}}\right]<0[143,144]$. Moreover, the Nernst signal increases dramatically with decreasing temperature - the effect that has been attributed to the superconducting fluctuations and/or fluctuating vortex pairs whose (positive) contribution dominates over the quasiparticle one (the latter can be of either sign, depending on the dominant type of carriers) upon approaching $T_{c}$. Besides, $v_{\mathrm{N}}$ turns out to be strongly affected by a proximity to the pseudogap regime and can even become anisotropic.

The 'Fermi-surfaced' solution (22) should be compared with the significantly more exotic one obtained in Refs. [136, 137]

$$
z=4 / 3, \Delta_{e}=-2 / 3, \Delta_{n}=2,
$$

where the thermoelectric coefficients $S \sim T^{1 / 2}, L_{\mathrm{H}} \sim T$, $v_{\mathrm{N}} \sim T^{-3 / 2}$ do not obey Eq. (23). Also, in this case an agreement with the linear behaviour of $L_{\mathrm{H}}$ reported in Refs. [143, 144] is guaranteed by imposing it as one of the constitutive relations. In spite of this predestined success, though, this scheme fails to reproduce the linear (up to a constant) thermopower, although it claimed the alternate $S=a-b T^{1 / 2}$ dependence to provide an even better fit to the data [142].

Also, the solution (24) predicts $C \sim T^{3 / 2}$ and, therefore, appears to be at obvious odds with the observed 
thermodynamic properties as well [135]. Moreover, it predicts the linear in $T$ (longitudinal) Lorenz ratio $L=\kappa / T \sigma$ similar to its Hall counterpart, contrary to a constant $L$, as suggested by the scenario (22).

Although an additional experimental effort is clearly called for in order to discriminate more definitively between the above predictions, it should be mentioned that more recently a slower-than-linear temperature behaviour of the (electronic) Lorenz ratio has been reported [145-147].

Lastly, the solution (24) features $\theta=0$ and strong (infrared) charge renormalization $\left(\Delta_{e}<0\right)$. Since in the presence of a well-defined $d$-dimensional Fermi surface the 'hyperscaling-violation' parameter is expected to coincide with its co-dimension, hence $\theta=d-1$ [47-54], the value $\theta=0$ hints at a point-like ('Dirac') Fermi surface (if any). In contrast, Eq. (22) suggests a rather simple physical picture where neither the Fermi liquid-like dispersion nor the effective charge demonstrate any significant renormalization. Thus, to construct a viable phenomenological description of the optimally doped cuprates one might be able to do away without introducing the additional charge exponent $\Delta$, contrary to the assertions made in Refs. [59, 136, 137, 148, 149.

It might also be worth mentioning that another long-time baffling, the underdoped, phase of the cuprates has recently been downgraded to a potentially simpler organized state of matter with some of its properties being Fermi-like [150-152].

Also, the recent attempt to match the exponents (24) with the predictions of the semi-phenomenological 'unparticles' model showed that it can only be achieved at the expense of a further, multi-flavoured, extension of that scenario [153]. However, the sheer complexity of the proposed construction seems to indicate rather clearly that the latter can hardly provide a natural (let alone, minimal) theory of the anomalous transport in the cuprates.

Moreover, in the last of Refs. [153-155] the method of fractional derivatives was employed to generalize the Maxwell's and continuity equations:

$$
\begin{aligned}
& \partial_{\mu}^{\alpha} F^{\mu v}=J^{v}, \\
& \partial_{i}^{\alpha} J^{i}+\partial_{0} \rho=0 .
\end{aligned}
$$

However, it can be readily seen that Eqs. (25) are only consistent when the dimension of the vector potential $\left[A_{i}\right]=\left[\partial_{i}^{\alpha}\right]=\alpha$ equals $z$ (which was chosen as $z=1$ in Refs. [153-155]), thus prohibiting it from taking any anomalous value.

In fact, a gradual realization of how strained can be the attempts to squeeze the holographic phenomenol- ogy of the cuprates into the 'Procrustes bed' of the twoparametric HV geometries may have already started making its way into the holographic community [156].

Another viable candidate for applications of the holographically-inspired scaling theory is provided by the self-consistent strong-coupling solutions aimed at describing the properties of certain $2 d$ and $3 d$ antiferromagnetic metals. Analyzed under the assumption of a momentum-independent NFL selfenergy, $\Sigma(\omega) \sim \omega^{1-\alpha}$, it yields the critical exponents as sole functions of the spatial dimension [157-159]

$$
\begin{aligned}
& \alpha=1 / 2-1 / z_{b}, z_{b}=4 d / 3, z_{f}=1 /(1-\alpha), \\
& v=1 /\left(2+z_{b} \alpha\right)
\end{aligned}
$$

Notably, the hyperscaling relations are still obeyed, resulting in the anomalous dependences:

$$
C \sim T^{1-\alpha}, \sigma \sim \omega^{\alpha-1}, \chi_{s} \sim T^{\alpha-1} .
$$

In the presence of disorder these results are likely to be modified, though [160-162].

Another recent work [163] utilized the functional RG technique, thus obtaining a different solution which might be pertinent to the AFM metals: $z_{f}=z_{b}=3 / 2, \theta=0, v=\gamma=1$. Reproducing these solutions and searching for the new ones [95] poses an interesting challenge and presents an important test for the holographically inspired scaling theory of these materials.

\section{From AdS/CMT to holographic transport}

A great many of the early works on CMT holography utilized the standard holographic recipe for computing electrical conductivity and other kinetic coefficients as boundary limits of the ratios between the convergent (associated with a response) and divergent (associated with a source) terms in the solutions of the radial classical equations $[1-6]$. This prescription would result in, e. g. the expression for the electrical conductivity

$$
\sigma(\omega)=\frac{1}{\mathrm{i} \omega} \frac{B}{A}=\left.\frac{1}{\mathrm{i} \omega} r^{2-d} \frac{\mathrm{d} \ln F_{r i}}{\mathrm{~d} r}\right|_{r \rightarrow 0},
$$

where the component of the field tensor $F_{r i}=A+B r^{d-1} /$ $(d-1)$ is a bulk dual of the boundary electric current.

Moreover, in the absence of any dilaton accounting for the scale dependent couplings and bringing about a dependence on the radial holographic coordinate, the physical observables become anomaly-free and scale-invariant, hence unaffected by renormalization. In that case they can be cast in a purely algebraic form 
in terms of the horizon metric, as per the 'membrane paradigm' 164-169]. For instance, the electrical conductivity and charge susceptibility read

$$
\begin{aligned}
& \chi=\left(\int_{0}^{\mathrm{h}} \mathrm{d} r \frac{g_{r r} g_{t t}}{(-g)^{1 / 2}}\right)^{-1}, \\
& \sigma=\left.\left(\frac{-g}{g_{r r} g_{t t}}\right)^{1 / 2} g^{i i}\right|_{r_{\mathrm{h}}},
\end{aligned}
$$

where $g$ is the determinant of the metric. Per the Einstein's relation the two are related via the diffusion coefficient, $\sigma=\chi_{c} D$.

However, while it might be possible to predict such general features as the exponents in the powerlaw frequency dependences of the optical electrical and thermal conductivities [130] by using the formal prescriptions similar to Eqs. $(\overline{28}, 29)$, the latter do not properly account for the actual physical contents of the boundary theory and a potentially intricate interplay between its different scattering mechanisms.

A list of the proposed sources of current and/or momentum relaxation (which is often confusingly referred to as 'dissipation') employed in holography includes random boundary and horizon potentials, helical (Bianchi VII -type) and spatially periodic ('Qlattice') geometries, massive gravity, axion fields, etc. [13, 68, 170-185.

Common to all the different approaches, though, is the unifying fact that the conductivity and other kinetic coefficients would be typically given by a sum of two terms,

$$
\sigma(\omega)=\sigma_{0}+\frac{e^{2} n^{2}}{\chi_{P P}(\gamma-\mathrm{i} \omega)}
$$

although in the 'top-down' constructions using the DBI action [9-13] Eq. (30) would be replaced by $\sigma=\sqrt{\sigma_{0}^{2}+\sigma_{1}^{2}}$.

In Eq. (30) the momentum susceptibility $\chi_{P P}=E+P$ is equal to the enthalpy density, and the first term corresponds to the (potentially, universal) diffusion-limited contribution to the conductivity that survives in the particle-hole symmetric limit of zero charge density $(n \rightarrow 0)$. It is often associated with the processes of 'pair creation' by which a neutral system develops a non-vanishing (yet, finite) conductivity in the absence of momentum relaxation $(\gamma \rightarrow 0)$, while the DC $(\omega \rightarrow 0)$ conductivity of a finite density system is governed by the second term in (30), becoming infinite in the limit $\gamma \rightarrow 0$.

Moreover, in the case of Q-lattices and $1 d$ periodic potentials (but not those that break translational in- variance in all the spatial dimensions) the conductivity can still be expressed in terms of the horizon data, thus generalizing the case of zero charge density and no momentum relaxation. Interestingly, though, even for generic ('non-Q') lattices which break translational symmetry in all the spatial dimensions the conductivity can be obtained by solving some linearized, time-independent, and forced Navier-Stokes-type equations of an effective (charged and incompressible) fluid living on the horizon [186-189], thus extending the 'membrane paradigm' along the lines of the general concept of a fluid-gravity correspondence [190-193]. The latter allows for a dual description of the bulk gravity theory in terms of the hydrodynamics of a certain boundary fluid whose stress-energy tensor acts as a source for the boundary metric. However, while unveiling yet another intrinsically geometric aspect of an underlying quantum dynamics, the fluid-gravity correspondence is clearly not identical to (albeit, possibly, far more general than) the original string-theoretical holography.

In certain axion models [194-200], the elastic rate can be readily computed although the resulting behaviour $\gamma \sim \max \left[T^{2}, m^{2}\right] / T$ can hardly remain physical in the $T \rightarrow 0$ limit. Remarkably, though, at $m=\sqrt{2} r_{\mathrm{h}}=\sqrt{8} \pi T$ the linearized (classical gravitational) equations of motion appear to be exactly solvable, yielding the frequency- and momentum-dependent response functions $G(\omega, k)$ in a closed form and also signalling an emergent $\operatorname{SL}(2, R) \times \operatorname{SL}(2, R)$ symmetry [194-200].

In the more general situation, momentum conservation would be broken by some operator $\mathcal{O}$ with the dimension $\Delta_{O}$ and the corresponding elastic scattering rate $\gamma$ can be expressed in terms of the spectral density $D(\omega, k)=\operatorname{Im}\langle\mathcal{O}(\omega, k) \mathcal{O}(-\omega,-k)\rangle[13,68$, 170-185]:

$$
\gamma=\left.\frac{1}{d \chi_{P P}} \int \mathrm{d} \mathbf{k} k^{2} \frac{\operatorname{Im} D(\omega, k)}{\omega}\right|_{\omega \rightarrow 0} .
$$

In the EMD context, the following non-universal behaviour of the DC conductivity was obtained by treating the pertinent 'random Ising magnetic field' disorder perturbatively [201-203]:

$$
\sigma(T) \sim T^{2\left(z-1-\Delta_{\mathcal{O}}\right) / z} .
$$

Also, the generalized Harris criterion for the relevance of disorder

$$
\Delta<(d-\theta) / 2+z
$$

gets saturated for $\Delta_{\mathcal{O}}=(d-\theta) / 2+z$, resulting in the universal, yet $\theta$-dependent, dependence 201-203. 


$$
\sigma(T) \sim T^{(\theta-2-d) / z} \sim \sim 1 /\left(\mathcal{S}^{2}\right),
$$

where the concomitant scaling of the entropy density, $\mathcal{S} \sim T^{(d-\theta) / z}$, has been taken into account. In Eq. (34) the second factor can be interpreted as the 'thermal graviton mass', $m \sim T^{1 / z}$. Thus, it is only in the extreme 'locally-critical' limit $z \rightarrow \infty$ where the famous prediction $\sigma \sim 1 / \mathcal{S}$ of Refs. [204, 205] could be confirmed without any sleight of hands.

Moreover, should the relevant bosonic modes happen to be transverse and, therefore, protected from developing a mass on the grounds of unbroken gauge invariance (as it would be the case in any SU(2)- or $\mathrm{U}(1)$-symmetric spin liquid state), the problem of computing the conductivity would require a full nonperturbative solution.

Notably, even in the HV model with $z=3 / 2$ and $\theta=1$ (which, for $d=2$, satisfy the relation $z=1+\theta / d$ saturating the first of Eqs. (7)) proposed as a viable candidate for the bulk dual of the theory of $2 d$ fermionic matter coupled to an overdampted bosonic (e. g. gauge) field [47-54, 206] the conductivity exponent in Eq. (34) $((\theta-2-d) / z=-2)$ would be different from its target value $(-1)$. This observation, too, might be pointing at some deeper problem with reproducing the ubiquitous linear normal state resistivity of the cuprates in the framework of the HV holographic models.

As far as the optical conductivity is concerned, in the last of Refs. [201-203] the asymptotic $\sigma(\omega) \sim \omega^{(2-\theta-d) / z}$ was obtained which does reproduce the desired behaviour (9) for the above $d, z$, and $\theta$. Notably, though, this exponent is different from the universal value, $(2-d)(1-\theta / d) / z$, that was independently obtained in a number of earlier works utilizing the HV geometries [130, 207-209].

More recently, the main focus of the CMT holographic phenomenology began to shift towards developing a general 'holography-augmented' transport theory [194-200, 210-222]. This commendable effort strives to establish general relations between the transport coefficients and find their bounds (if any) that could remain valid regardless of the (in)applicability of the generalized holographic conjecture itself. Such relations are expected to hold in the hydrodynamic regime governed by strong inelastic interactions where the rates of momentum relaxation due to elastic disorder, lattice-assisted inelastic (Umklapp) electron-electron and electron-phonon (outside of the phonon drag regime) scattering are all much smaller than the universal inelastic rate $\Gamma \sim T$ of the normal collisions controlling the formation of a thermalized hydrodynamic state itself [82, 83, 204, 205].

Notably, the onset of hydrodynamics is a distinct property of the strongly correlated systems, as it is unattainable in the standard FL regime where all the local (in the $k$-space) quasiparticle densities $n_{k}$ are nearly conserved. The hydrodynamic regime does not set in for $d=1$ either due to the peculiar $1 d$ kinematics which facilitates the emergence of infinitely many (almost) conserved densities.

In the hydrodynamic regime, the holographic results have also been systematically compared to those of the hydrodynamic [210-217] and memory matrix [218-222] formalisms which do not rely on the existence of well-defined quasiparticles. The kinetic coefficients obtained by means of these alternate techniques appear to be similar to the holographic expressions (30), identifying the density $n$ with the current-momentum susceptibility $\chi_{J P}=\langle J \mid P\rangle=n$ which quantifies the contribution to the current from 'momentum drag'. Such formalism provides an elegant and physically transparent language for discussing, e. g. a decoupling of the electric current onto the coherent component parallel (in the functional sense) to the momentum $P$ and the orthogonal, incoherent, one which has no overlap with $P$ and is responsible for the universal conductivity of the neutral (particle-hole symmetric) system:

$$
J=\frac{\chi_{J P}}{\chi_{P P}} P+J_{\text {incoh }} .
$$

Although under a closer inspection the hydrodynamic results were found to be subtly different (beyond the leading order) from the earlier holographic ones, such differences have been reconciled by including the corrections to $\sigma_{o}$ and the residue of the pole in Eq. (30) at $\omega=-\mathrm{i} \gamma$ ('Drude weight') [194-200, 210217]. This agreement between the holographic and hydrodynamic/memory matrix analyses was argued to provide an additional evidence supporting (at least, the transport-related aspects of) the former.

It should be noted, though, that the AC kinetic coefficients akin to Eq. (30) appear to have a strictly monotonic frequency dependence which is governed by the coherent zero-frequency Drude peak whose presence reflects the existence of a (nearly) conserved momentum which overlaps with the electrical current for $n \neq 0$.

On the other hand, a non-monotonic - or, else, strictly universal (frequency-independent) - behaviour would be necessary in order for the conductivity to comply with the holographic sum rules proposed in Refs. [30-34]:

$$
\begin{aligned}
& \int_{0}^{\infty}(\sigma(\omega)-\sigma(\infty)) \mathrm{d} \omega=0, \\
& \int_{0}^{\infty}\left(\frac{1}{\sigma(\omega)}-\frac{1}{\sigma(\infty)}\right) \mathrm{d} \omega=0 .
\end{aligned}
$$


These sum rules account for a transfer of the spectral weight from the coherent Drude peak to the incoherent high-frequency tail. Such a non-monotonic behaviour develops at $\omega \sim \Gamma$ and may or may not be detectable by the hydrodynamic/memory matrix analyses, though.

One of the most striking predictions of the memory matrix calculations is that of the reported absence of (many-body) localization and diffusion-dominated metallic transport in strongly interacting 'strange metals' 223-226]. It would seem rather surprising, though, that while imposing robust conductivity bounds in $d=1$ and 2 (where in the latter case the lower bound even seems comparable to the upper one), this approach fails to find such bounds in $d>2$ where, according to the general wisdom, localization would be even less likely to set in.

At a deeper level, a fully quantum description of localization (or a lack thereof) still seems to remain out of reach in all the existing holographic treatments of disorder. In fact, any 'mean-field' approximation is likely to be in principle incapable of taking into account not only the localization-related phenomena (multiple acts of elastic scattering) but also the interference ones (multiple acts of consecutive elastic and inelastic scattering).

For one thing, the former type of the conductivity corrections would depend not only on the elastic scattering rate but also on some rather special inelastic ones (Cooperon/Diffuson phase-breaking), whereas the latter would typically acquire its temperature dependence via the fermion occupation factors. Neither source of the $T$-dependence can be readily envisioned in the variational approach of Refs. [223-226], though. While suitable for analyzing classical percolation, this approach operates in terms of the Kirchoff's law for a random network of classical resistors and employs the quadratic Thomson's variational action akin to that for maximum entropy production. In light of such potential caveats it does not seem surprising that the results of Refs. [223-226] suggest the diffusive metallic behaviour even at weak repulsive interactions strengths in $2 d$ - which would then be in conflict even with the perturbative Altshuler-Aronov theory.

Leaving out the remaining possibility of finding a better variational ansatz that could yield a lower conductivity than the simplest one of a constant current/density employed in Refs. [223-226] and resulting in the above lower bound, it is worth noting that the scenario of Refs. [223-226] might also be limited to the rather special class of models where no coupling between the translation symmetry-breaking (Stueckelberg) degrees of freedom and the Maxwell field is allowed.
To that end, a further investigation into the issue 224-227] showed that, in contrast, no finite (let alone, universal) lower bound seems to exist in the more general models which include generic couplings between the axions with the expectation values $\Phi_{i} \sim x_{\mu} \delta_{i}^{\mu}$ and vector and/or scalar fields:

$$
\Delta L=\sum_{n=1}^{\infty}\left(g^{\mu v} \partial_{\mu} \Phi_{i} \partial_{v} \Phi_{i}\right)^{n}\left[a_{n} F_{\mu v}^{2}+b_{n} \phi^{2}\right] .
$$

Thus, conceivably, the scenario of 'many-body delocalization' proposed in Refs. [223-226] is neither generic nor generalizable beyond the scopes of the variational analysis. However, the results of Refs. [224-229], too, should be taken cautiously, as, e. g. the second reference finds the conductivity to be potentially negative, depending on the strength of the couplings $a_{n}, b_{n}$. Also, in the last of Refs. [224-229] predicting a seemingly realistic insulator-superconductor transition in the framework of the 'anti-DBI' theory (whose generalized Maxwell action contains the $\left(F_{\mu \nu} F^{\mu v}\right)^{2}$ correction with a positive sign, opposite of that in the DBI action) the collective sound modes become super-luminal, as their speed exceeds the (effective) speed of light.

In that regard it should be mentioned that, although these and other predictions would certainly benefit from clear disclaimers about the limited range of the holographic parameters for which physically sensible predictions (e. g. positive conductivity) can be made, conspicuously, such limits do not readily follow from the phenomenological 'bottom-up' approach itself. In particular, it would be rather premature to apply any of the aforementioned holographic scenaria to the analysis of, e. g. the 2DEG systems demonstrating the apparent metal-insulator transition, complete with its peculiar scaling properties [230-233].

\section{Holographic transport: Dirac/Weyl materials}

An important example of the systems whose transport properties can be studied systematically and then compared to the holographic predictions is provided by the interacting Dirac/Weyl (semi)metals. In the past, the transport properties of (pseudo)relativistic systems have already been addressed in the context of, both, interacting $2 d$ bosons and fermions [234-236]. Such studies utilized the standard method of quantum kinetic equation which offers a viable insight that can be compared to the specific predictions of the general theory of holographic transport. The fermionic variant of this problem pertinent to the case of Coulomb-interacting graphene was studied in the so-called two-mode approximation where 
the emphasis was made on the conservation of charge and energy densities, as well as momentum density which is equivalent to the energy current up to the higher-order dissipative corrections (a feature that is specific to the (pseudo)relativistic dispersion) [237-239].

In the case of a weak Coulomb coupling $\alpha=e^{2} /$ $h v \ll 1$ the equilibration rate of all other, non-conserved, modes would be of order $\Gamma_{\text {non-cons }} \sim \alpha^{2}|\ln \alpha| T$ which is higher than that of the conserved ones, $\Gamma_{\text {cons }} \sim \alpha^{2} T$, thanks to the $2 d$ kinematic (logarithmic) divergence of the Coulomb collision integral. Thus, the conserved modes can be singled out, while neglecting all those that undergo a faster relaxation 237-239.

It turns out, however, that the system possesses another (nearly) conserved density which is the imbalance' mode corresponding to the total number of electrons and holes (as opposed to their difference related to the charge density) [240-247]. Away from the neutral (particle-hole symmetric) regime its relaxation takes place through the Auger-type processes with the still slower rate of order $\Gamma_{\text {imb }} \sim T^{4} / \mu^{3}$. Close to the neutrality point the results of the two- and three-mode analyses appear to differ somewhat, thus showing that the imbalance mode can indeed impact the low-energy hydrodynamic behaviour [240-247].

It would, therefore, be desirable to carry out the three-mode calculations for the $\mathrm{SU}(N)$-symmetric fermions with $N \gg 1$, focusing on the regime $1 / N \ll \alpha^{2} \ll 1$ where a comparison with the holographic predictions can be made [95].

Notably, the kinetic equation tends to yield the conductivity which develops the Drude peak followed by a dip at $\omega \sim \Gamma$, while no other peaks (nor zeros) emerge, in contrast to the holographic/hydrodynamic/memory matrix result (30). Besides, it remains to be seen as to how the explicitly computed corrections to the optical conductivity of graphene [247-253]

$$
\frac{\sigma(\omega)}{\sigma(\infty)}=1+\frac{C}{|\ln \omega|}
$$

match with the general prediction 86$]$

$$
\frac{\sigma(\omega)}{\sigma(\infty)}=1+\sum_{n} C_{n}\left(\frac{T}{\omega}\right)^{\Delta},
$$

where the sum is taken over the operator expansion of the product of two current operators.

Another quantity of special interest would be the Lorenz ratio (ordinary, as well as the Hall one) as a function of frequency. In the DC limit and near the neutrality point this ratio is dramatically enhanced because the heat and charge currents are controlled by two different mechanisms: momentum relaxation vs electron-hole pair creation. This prediction was found to be in a good agreement with the recent data on graphene [254-256] showing a 20-fold enhancement (although the ratio $\eta / s \sim 10$ estimated in Refs. [254-256] seems to indicate that even a free-standing graphene may not be that strongly coupled, after all).

It would also be interesting to generalize the analysis to $d=2+\epsilon$ dimensions where the effects of the Coulomb interactions are expected to be less dramatic and the separation between the slowly vs rapidly decaying modes becomes less pronounced [95].

Besides, one would need to introduce yet another nearly conserved density describing an imbalance between the numbers of quasiparticles in the vicinity of the two different Weyl points. This 'chiral' density incorporating (for $\epsilon=1$ ) the effects of the $3 d$ chiral anomaly gives rise to such concomitant transport phenomena as a negative magnetoresistance [257-259]. Some of its underlying geometric aspects can be observed even at the semiclassical level where the phase-space dynamics is naturally described in terms of the Bloch curvature [124-126] $\Omega_{k}=\epsilon_{i j}\left\langle\frac{\partial \Psi}{\partial k_{i}} \mid \frac{\partial \Psi}{\partial k_{j}}\right\rangle$ which enters the semiclassical equations of motion as a dual of the physical magnetic field:

$$
\begin{aligned}
& \frac{\mathrm{d} \mathbf{r}}{\mathrm{d} t}=\frac{\partial \epsilon}{\partial \mathbf{k}}+\Omega_{\mathbf{k}} \times \frac{\mathrm{d} \mathbf{k}}{\mathrm{d} t}, \\
& \frac{\mathrm{d} \mathbf{k}}{\mathrm{d} t}=-\frac{\partial \epsilon}{\partial \mathbf{r}}+\mathbf{B} \times \frac{\mathrm{d} \mathbf{r}}{\mathrm{d} t} .
\end{aligned}
$$

The current relaxation is then described by the equations

$$
\begin{aligned}
& \partial_{v} T^{i v}=F^{i v} J_{v}-\gamma T^{i t}, \\
& \partial_{v} J^{v}=\alpha \epsilon_{\mu \nu \lambda \rho} F^{\mu v} F^{\lambda \rho} .
\end{aligned}
$$

Reproducing these effects in their entirety would provide another check point for (and further advance) the holographic transport theory [260, 261].

In that regard, one should also mention a series of works aimed at connecting holography to the more conventional field theoretical analysis [262-265]. It seems, though, that the actual graphene and $3 d$ Weyl/ Dirac metals alike can hardly provide a viable playground for the CMT holography, as the effects of interactions in these materials are either mild or even outright weak. It would, therefore, be quite interesting to find some realization of their counterparts 'on steroids' in the condensed matter realm. 
The extended multi-mode kinetic analysis of graphene may also allow for a deeper understanding of the role of viscosity in electron transport. The viscous terms become relevant for $\eta>(e n w)^{2} / \sigma$ where $w$ is the width of the graphene sample and such analysis can even be performed in the framework of the Navier-Stokes equation, this time in real space [266], with predictions consistent with the data of Refs. [254-256. Incidentally, this regime has recently been studied in the ultra-pure $2 d$ metal $\mathrm{PdCoO}_{2}$ [267], although the estimated ratio $\eta / S \sim 10^{6}$ would hardly allow one to view it as ideal fluid.

Also, the problem of a hot spot relaxation in graphene [240-247] presents a specific example of the general 'quench'-type phenomena which, too, have been extensively addressed by the CMT holography [268-274]. The $1 d$ variant of this problem involves two reservoirs of different temperatures $T_{L}$ and $T_{R}$ which are brought into a thermal contact, its solution featuring a non-equilibrium stationary state characterized by a definite temperature $\left(T_{L} T_{R}\right)^{1 / 2}$ and constant energy flux (this solution does not seem to readily extend to the limit of either vanishing temperature, though). Moreover, this stationary state was shown to spread outward in the form of shock waves and was related to the Lorentz-boosted blackhole metric in the bulk, thus suggesting yet another explicit holographic connection.

To that end, it was conjectured in Refs. [268-274] that a similar behaviour would occur in higher dimensions as well. However, the recent analyses of the hot spot relaxation in $2 d$ graphene [240-247] suggest that a more involved scenario where, both, the resistive and viscous effects interfere with one another might be realized. Notably, the recent holographic work of Refs. [275, 276], too, shows a more involved dynamics of the expanding stationary state.

Further studies of these topics are strongly warranted, also in light of the possibility to test some of the proposed hallmarks of the hydrodynamics behaviour, such as the specific relations between linear and non-linear transport [224-229], new (NFL) hydrodynamic modes [210-217], and sound-wave resonances in finite geometries [254-256].

\section{Analogue holography demonstrators}

The above examples of 'emergent geometry' prompt a systematic quest into the apparent holography-like relationships that would be governed by the already known, rather than some hypothetical, physics. To that end, the general holographic concept can benefit from the possibility of being simulated in various controlled 'analogue' environments.
One prospective design of a 'holography simulator' was proposed for implementation in flexible graphene and other semi-metallic monolayers (silicene, germanene, stanene, etc.) [277]. Such stress-engineered desktop realizations of the system of $2 d$ Dirac fermions in a curved geometry [278-284] can also be grown on commensurate substrates (e. g. $h-\mathrm{BN}$ ) in order to endow the bulk fermions with a finite mass via hybridization.

In Ref. [277] a number of situations were discussed where a physical edge of the curved graphene flake which supports (almost) non-interacting massive Dirac fermions propagating in a curved $2 d$ space exhibits a behaviour that would be typically attributed to the effects of some phantom $1 d$ interactions. In contrast to the aforementioned 'semi-local' scenario of Refs. [3544 , though, it is the momentum dependence of the $1 d$ propagator $G(\omega, k)$ that tends to become non-trivial.

Specifically, in graphene the artificial gauge and metric fields represent the elastic (phonon) degrees of freedom, their effective vector and scalar potentials

$$
A_{0} \sim u_{x x}+u_{y y}, A_{x} \sim u_{x x}-u_{y y}, A_{y} \sim u_{x y}
$$

being composed of the components of the strain tensor [285-293], $u_{i j}=\frac{1}{2}\left(\partial_{i} u_{j}+\partial_{j} u_{i}+\partial_{i} h \partial_{j} h\right)$.

Here $u_{i}(\mathbf{x})$ and $h(\mathbf{x})$ are the local in- and out-ofplane displacements of the monolayer, respectively, while the valley-specific fields $A_{\mu}$ have opposite signs at the two different Dirac points. Computing the massive free Dirac fermion propagator in a curved space and taking its boundary limit one obtains the largescale asymptotic behaviour (4) governed by the geodesic action $S(\tau, x)$ for the background metric. For instance, in the case of a graphene flake shaped as a surface of rotation with the line element (here $r$ and $\phi$ are the normal polar coordinates)

$$
\mathrm{d} l^{2}=d r^{2}\left[1+\left(\frac{\partial h(r)}{\partial r}\right)^{2}\right]+r^{2} \mathrm{~d} \phi^{2}
$$

with $h(r) \sim(R / r)^{\eta}$ for $r \geq a$ the 'warp factor' $g(r) \sim 1 / r^{2 n+2}$ diverges at small $r$. One then obtains the geodesic action

$$
S(\tau, x)=m \sqrt{\tau^{2}+\left(R x^{\eta}\right)^{2 /(\eta+1)}},
$$

which reveals an unconventional behaviour of the boundary propagator as a function of the distance along the edge, thus suggesting the 'holographic' dynamical critical exponent $z=\eta /(\eta+1)$.

It is instructive to compare the asymptotic (4) with the action given by (44) with the propagator of $1 d$ fermions interacting via a pairwise potential $U(x) \sim 1 / x^{\zeta}$ where $\zeta<1$. Using the standard $1 d$ bosonization 
technique and matching the large- $x$ asymptotics, one finds that (44) can mimic the spatial decay of the $1 d$ propagator in the presence of such interactions, provided that $\eta=(1-\zeta) /(1+\zeta)$.

Another instructive example is provided by the line element

$$
\mathrm{d} l^{2}{ }_{\log }=\mathrm{d} r^{2}+R^{2} \exp \left(-2(r / R)^{\lambda}\right) \mathrm{d} \phi^{2},
$$

in which case the geodesic action reads

$$
S(\tau, x)=m \sqrt{\tau^{2}+R^{2}(\ln x / a)^{2 / \lambda}} .
$$

For $\lambda=1$ and at large $x$ the propagator decays algebraically, $G(0, x) \sim 1 / x^{m R}$ which is reminiscent of the behaviour found in the $1 d$ Luttinger liquids, while for $\lambda \neq 1$ Eq. (46) yields a variety of stretched/compressed exponential asymptotics which decay faster (for $\lambda<1$ ) or slower (for $\lambda>1$ ) than any power-law. For instance, by choosing $\lambda=2 / 3$ one can simulate a faster-thanalgebraic spatial decay, $G(0, x) \sim \exp \left(-\right.$ const $\left.\ln ^{3 / 2} x\right)$, in the $1 d$ Coulomb gas $(\sigma=1)$ which is indicative of the incipient $1 d$ charge density wave state.

For other values of $\lambda$ Eq. (46) reproduces the behaviour in the boundary theory governed by the interaction $U(x) \sim(\ln x)^{(2 / \lambda)-3 / x}$. Although the physical origin of such a bare potential would not be immediately clear, multiplicative logarithmic factors do routinely emerge in those effective $1 d$ interactions ('double trace operators') that are associated with various marginally (ir)relevant two-point operators. On the experimental side, the boundary correlations can be probed with such established techniques as time-of-flight, edge tunneling, and local capacitance measurements, thus potentially helping one to hone the proper analogue-holographic 'dictionary'.

This holography-like correspondence once again suggests that some limited form of a bulk-boundary relationship might, in fact, be quite robust and hold regardless of whether or not the systems in question possess any particular symmetries, unlike in the original AdS/CFT construction. Naively, this form of correspondence can even be related to the Einstein's equivalence principle (i. e. 'curvature equals force'), according to which free motion in a curved space should be indistinguishable from the effect of a physical interaction (only, this time around, in the tangential direction).

As an alternate platform for doing analogue holography, optical metamaterials have long been considered as candidates for simulating such effects of general relativity as event horizons, redshift, black, white, and worm holes, inflation, Hawking radiation, dark energy, multiverse, Big Bang and Rip, metric signatures transitions, 'end-of-time', and other cosmological scenaria.

However, the earlier proposals focused on the effective 3+1-dimensional metrics which requires some intricate engineering of the locked permittivity $(\epsilon)$ and permeability $(\mu)$ tensors [294-302]:

$$
\epsilon^{i j}=\mu^{i j}=g^{i j} \sqrt{-g} /\left|g_{00}\right| .
$$

More recently, it was proposed to use extraordinary (TM-polarized) monochromatic photons with the dispersion $\omega^{2}=k_{z}^{2} / \epsilon_{x y}+k_{x y}^{2} / \epsilon_{z z}$ for simulating the effective $2+1$-dimensional metrics

$$
g_{\tau \tau}=-\epsilon_{x y}, g_{r r}=g_{\phi \phi} / r^{2}=-\epsilon_{z z}
$$

in the media with $\mu=1$ [303-311].

In the hyperbolic regime, $\epsilon_{x y}>0$ and $\epsilon_{z z}<0$, the momentum component $k_{z}$ can then be thought of as an effective frequency, whereas $\omega$ plays the role of mass. In Ref. [312] it was shown that the experimentally attainable two-component metamaterial configuration consisting of alternating metallic and insulating (flat or cylindrical) layers can simulate some of the HV geometries. The boundary propagator describing static spatial correlations of the optical field on the interface between the metamaterial and vacuum was found to behave as

$$
\left\langle E_{\omega}(\mathbf{x}) E_{-\omega}(0)\right\rangle \sim \exp \left[-|\omega \mathbf{x}|^{\theta / z}\right],
$$

which should be contrasted to its counterpart in an isotropic medium with a (negative) dielectric constant, $\left\langle E_{\omega}(\mathbf{x}) \mathrm{E}_{-\omega}(0)\right\rangle \sim \exp (-\omega|\mathbf{x}|)$. Experimentally, such correlations can be studied by analyzing the statistics of a non-local optical field distribution with the use of holographic and speckle interferometry. Albeit still requiring a careful engineering of the dielectric media, some hallmark features of the analogue holography could, in principle, be detected and then compared with, e. g. correlators of the vertex operators in the theory of a strongly selfinteracting $2 d$ bosonic field, akin to that describing the thermodynamics of a fluctuating elastic membrane.

Lastly, one could further elaborate on yet another (historically, far more extensively studied) potential playground for analogue holography which is provided by the acoustic realizations of 'emergent gravity' [313-316]. By implementing such proposals one might also hope to establish a putative bulk-boundary correspondence (alongside its own 'dictionary') for a broader variety of the combined (pseudo)gravitational backgrounds, thereby gaining a better insight 
into the physical origin(s) of the apparent holographylike properties of various CMT and AMO systems.

\section{Conclusions}

This critical discussion demonstrates that, despite the widespread expectations and reassuring declarations on some part of the holographic community, the CMT holography still lacks a true 'smoking gun' that could lend a firm support for this approach (albeit, possibly, in some reduced, rather than its most broad, overreaching, form). Therefore, it is perhaps not too surprising that there is still no consensus, neither on the exact implications of the reports of some apparent agreement between the holographic predictions and the results of other approaches and/or experimental data, nor the general applicability and the principal limitations of the holographic approach itself.

Together with the systematic comparison between the predictions of the CMT holography and other, more traditional, approaches and/or experimental data it would be a necessary step towards vetting the intriguing, yet speculative, holographic ideas before the latter can be rightfully called a novel technique for tackling the 'strange metals' and other strongly correlated materials. To prove genuinely useful the holographic theory needs to come up with detailed and unambiguous predictions that would be distinguishable from those of the general hydrodynamic approach which does not necessarily require any specific references to holography.

As to the general justification for the applied holography, it remains to be seen whether a viable holographic framework can finally emerge out of the RG procedure on the tensor network states, hydrodynamic fluid/gravity duality, algebra of bi-linear boundary operators, or any combination thereof. The very multitude of the currently available proposals may seem to attest to the potential existence of some rather general, intrinsically geometrical, principles that govern a broad variety of the strongly-correlated many-body systems, albeit not necessarily copying ad verbum all the machinery of the very specific original stringtheoretical construction. Thus, in any event, it would seem rather unlikely that a hands-on expertise in string theory should ever become a mandatory prerequisite for, e. g. understanding the cuprates.

The author gratefully acknowledges the support from and/or hospitality at the Aspen Center for Physics (funded by the NSF under Grant 1066293), the Galileo Galilei Institute (Florence), the Kavli Institute for the Physics and Mathematics of the Universe (Kashiwa), the Kavli Institute for Theoretical Physics (Beijing), the International Institute of Physics (Na- tal), and NORDITA (Stockholm) during the various stages of compiling this review.

\section{References}

[1] J. Polchinski, Introduction to gauge/gravity duality, http://arxiv.org/abs/1010.6134

[2] J. McGreevy, Holographic duality with a view toward many-body physics, Adv. High Energy Phys. 2010, 723105 (2010).

[3] S.A. Hartnoll, Lectures on holographic methods for condensed matter physics, Classical Quant. Grav. 26, 224002 (2009).

[4] C.P. Herzog, Lectures on holographic superfluidity and superconductivity, J. Phys. A 42, 343001 (2009).

[5] S. Sachdev, What can gauge-gravity duality teach us about condensed matter physics? Annu. Rev. Cond. Matt. Phys. 3, 9 (2012).

[6] S. de Haro, D.R. Mayerson, and J. Butterfield, Conceptual aspects of gauge/gravity duality, http:// arxiv.org/abs/1509.09231

[7] M. Ammon and J. Erdmenger, Gauge/Gravity Duality: Foundations and Applications (Cambridge University Press, 2015).

[8] J. Zaanen, Y. Liu, Y.-W. Sun, and K. Schalm, Holographic Duality in Condensed Matter Physics (Cambridge University Press, 2015).

[9] S. Kobayashi, D. Mateos, S. Matsuura, R.C. Myers, and R.M. Thomson, Holographic phase transitions at finite baryon density, I. High Energy Phys. 2007(02), 016 (2007), https://arxiv.org/abs/hepth/0611099

[10]A. Karch and A. O'Bannon, Metallic AdS/CFT, J. High Energy Phys. 2007(09), 024 (2007), http:// arxiv.org/abs/0705.3870

[11] S. Kachru, A. Karch, and S. Yaida, Holographic lattices, dimers and glasses, Phys. Rev. D 81, 026007 (2010), http://arxiv.org/abs/0909.2639

[12] S.A. Hartnoll, J. Polchinski, E. Silverstein, and D. Tong, Towards strange metallic holography, J. High Energy Phys. 2010(04), 120 (2010), http:// arxiv.org/abs/0912.1061

[13] S. Ryu, T. Takayanagi, and T. Ugajin, Holographic conductivity in disordered systems, J. High Energy Phys. 2011(04), 115 (2011), http://arxiv.org/ abs/1103.6068

[14] S.-S. Lee, Quantum renormalization group and holography, J. High Energy Phys. 2014(01), 076 (2014).

[15]S.-S. Lee, Background independent holographic description: From matrix field theory to quantum gravity, J. High Energy Phys. 2012(10), 160 (2012).

[16] S.-S. Lee, Holographic description of large N gauge theory, Nucl. Phys. B 851, 143 (2011).

[17] S.-S. Lee, Holographic description of quantum field theory, Nucl. Phys. B 832, 567 (2010). 
[18] P. Lunts, S. Bhattacharjee, J. Miller, E. Schnetter, Y.B. Kim, and S.-S. Lee, Ab initio holography, J. High Energy Phys. 2015(08), 107 (2015).

[19] C.H. Lee and X.-L. Qi, Exact holographic mapping in free fermion systems, http://arxiv.org/ abs/1503.08592

[20] M. Miyaji and T. Takayanagi, Surface/state correspondence as a generalized holography, http:// arxiv.org/abs/1503.03542

[21]Y. Nakayama, $a-c$ test of holography vs quantum renormalization group, http://arxiv.org/ abs/1401.5257

[22]S. Jackson, R. Pourhasan, and H. Verlinde, Geometric RG flow, http://arxiv.org/abs/1312.6914

[23] E. Kiritsis, W. Li, and F. Nitti, Holographic RG flow and the quantum effective action, Fortschr. Phys. 62(5-6), 389 (2014).

[24]S.-S. Lee, Horizon as critical phenomenon, http:// arxiv.org/abs/1603.08509

[25] S. Sachdev, Bekenstein-Hawking entropy and strange metals, Phys. Rev. X 5, 041025 (2015), http://arxiv.org/abs/1506.05111

[26] W. Fu and S. Sachdev, Numerical study of fermion and boson models with infinite-range random interactions, http://arxiv.org/abs/1603.05246

[27]A. Kitaev [unpublished].

[28] A. Jevicki, K. Suzuki, and J. Yoon, Bi-Local Holography in the SYK Model, http://arxiv.org/ abs/1603.06246

[29] J. Maldacena and D. Stanford, Comments on the Sachdev-Ye-Kitaev model, http://arxiv.org/ abs/1604.07818

[30]W. Witczak-Krempa, E. Sorensen, and S. Sachdev, The dynamics of quantum criticality via Quantum Monte Carlo and holography, Nat. Phys. 10, 361 (2014).

[31] W. Witczak-Krempa, Quantum critical charge response from higher derivatives: is more different? Phys. Rev. B 89, 161114 (2014).

[32]E. Katz, S. Sachdev, E.S. Sorensen, and W. WitczakKrempa, Conformal field theories at non-zero temperature: operator product expansions, Monte Carlo, and holography, http://arxiv.org/abs/1409.3841

[33] S. Bai and D.-W. Pang, Holographic charge transport in $2+1$ dimensions at finite $\mathrm{N}$, http://arxiv. org/abs/1312.3351

[34] K. Chen, L. Liu, Y. Deng, L. Pollet, and N. Prokof'ev, Universal conductivity in a two-dimensional superfluid-to-insulator quantum critical system, Phys. Rev. Lett. 112, 030402 (2013).

[35] S.S. Lee, A non-Fermi liquid from a charged black hole: A critical Fermi ball, Phys. Rev. D 79, 086006 (2009).

[36]H. Liu, J. McGreevy, and D. Vegh, Non-Fermi liquids from holography, Phys. Rev. D 83, 065029 (2011).

[37] N. Iqbal and Hong Liu, Real-time response in AdS/CFT with application to spinors, Fortsch.
Phys. 57, 367 (2009), http://dx.doi.org/10.1002/ prop.200900057

[38] M. Cubrovic, J. Zaanen, and K. Schalm, String theory, quantum phase transitions and the emergent Fermi-liquid, Science 325, 439 (2009).

[39] T. Faulkner, H. Liu, J. McGreevy, and D. Vegh, Emergent quantum criticality, Fermi surfaces, and AdS2, Phys. Rev. D 83, 125002 (2011).

[40] N. Iqbal, H. Liu, M. Mezei, and Q. Si, Quantum phase transitions in holographic models of magnetism and superconductors, Phys. Rev. D 82, 045002 (2010).

[41]T. Faulkner and J. Polchinski, Semi-holographic Fermi liquids, J. High Energy Phys. 2011(06), 012 (2011).

[42] N. Iqbal, H. Liu, and M. Mezei, Semi-local quantum liquids, http://arxiv.org/abs/1105.4621

[43] N. Iqbal, H. Liu, and M. Mezei, Quantum phase transitions in semi-local quantum liquids, https:// arxiv.org/abs/1108.0425

[44] R.G. Leigh, A.C. Petkou, and P.M. Petropoulos, Holographic three-dimensional fluids with nontrivial vorticity, http://arxiv.org/abs/1108.1393

[45] Q. Si and F. Steglich, Heavy fermions and quantum phase transitions, Science 329, 1161 (2010).

[46] D. Vollhardt, K. Byczuk, and M. Kollar, in: Strongly Correlated Systems, Springer Series in Solid-State Sciences, Vol. 117, eds. A. Avella and F. Mancini (Springer, 2011) pp. 203-236.

[47] S. Sachdev, Holographic metals and the fractionalized Fermi liquid, Phys. Rev. Lett. 105, 151602 (2010).

[48] L. Huijse and S. Sachdev, Fermi surfaces and gaugegravity duality, http://arxiv.org/abs/1104.5022

[49] S. Sachdev, A model of a Fermi liquid using gaugegravity duality, Phys. Rev. D 84, 066009 (2011).

[50] L. Huijse, S. Sachdev, and B. Swingle, Hidden Fermi surfaces in compressible states of gaugegravity duality, http://arxiv.org/abs/1112.0573

[51] B. Swingle, L. Huijse, and S. Sachdev, Entanglement entropy of compressible holographic matter: Loop corrections from bulk fermions, http://arxiv.org/ abs/1308.3234

[52]N. Ogawa, T. Takayanagi, and T. Ugajin, Holographic Fermi surfaces and entanglement entropy, J. High Energy Phys. 2012(01), 125 (2012).

[53]B. Swingle, Entanglement renormalization and holography, http://arxiv.org/abs/0905.1317

[54] B. Swingle, Constructing holographic spacetimes using entanglement renormalization, http://arxiv. org/abs/1209.3304

[55] S. Kachru, X. Liu, and M. Mulligan, Gravity duals of Lifshitz-like fixed points, Phys. Rev. D 78, 106005 (2008).

[56] N. Iizuka, N. Kundu, P. Narayan, and S.P. Trivedi, Holographic Fermi and non-Fermi liquids with transitions in dilaton gravity, http://arxiv.org/ abs/1105.1162 
[57]C. Charmousis, B. Goutéraux, B.S. Kim, E. Kiritsis, and R. Meyer, Effective holographic theories for low-temperature condensed matter systems, J. High Energy Phys. 2010(11), 151 (2010).

[58]B. Goutéraux, J. Smolic, M. Smolic, K. Skenderis, and M. Taylor, Holography for Einstein-Maxwelldilaton theories from generalized dimensional reduction, J. High Energy Phys. 2012(01), 089 (2012).

[59]B. Goutéraux and E. Kiritsis, Generalized holographic quantum criticality at finite density, J. High Energy Phys. 2011(12), 036 (2011).

[60]X. Dong, S. Harrison, S. Kachru, G. Torroba, and $\mathrm{H}$. Wang, Aspects of holography for theories with hyperscaling violation, http://arxiv.org/ abs/1201.1905

[61] E. Perlmutter, Hyperscaling violation from supergravity, http://arxiv.org/abs/1205.0242

[62] S.A. Hartnoll and A. Tavanfar, Electron stars for holographic metallic criticality, Phys. Rev. D 83, 046003 (2011).

[63] S.A. Hartnoll, D.M. Hofman, and D. Vegh, Stellar spectroscopy: Fermions and holographic Lifshitz criticality, http://arxiv.org/abs/1105.3197

[64] S. Sachdev, A model of a Fermi liquid using gaugegravity duality, Phys. Rev. D 84, 066009 (2011).

[65] G.T. Horowitz, J.E. Santos, and D. Tong, Optical conductivity with holographic lattices, https://arxiv.org/abs/1204.0519

[66] G.T. Horowitz, J.E. Santos, and D. Tong, Further evidence for lattice-induced scaling, http://arxiv. org/abs/1209.1098

[67] Y. Ling, C. Niu, J.-P. Wu, and Z.-Y. Xian, Holographic lattice in Einstein-Maxwell-dilaton gravity, J. High Energy Phys. 2013(11), 006 (2013), http://arxiv.org abs/1309.4580

[68] D. Vegh, Holography without translational symmetry, http://arxiv.org/abs/1301.0537

[69]D.N. Basov, R.D. Averitt, D. van der Marel, M. Dressel, and K. Haule, Electrodynamics of correlated electron materials, Rev. Mod. Phys. 83, 471 (2011).

[70]A. Donos and J.P. Gauntlett, Holographic Q-lattices, http://arxiv.org/abs/1311.3292

[71] K.-Y. Kim, K.K. Kim, Y. Seo, and S.-J. Sin, Coherent/incoherent metal transition in a holographic model, http://arxiv.org/abs/1409.8346

[72] B.W. Langley, G. Vanacore, and P.W. Phillips, Absence of power-law mid-infrared conductivity in gravitational crystals, J. High Energy Phys. 2015(10), 163 (2015).

[73]J. Erdmenger, P. Kerner, and S. Müller, Towards a holographic realization of Homes' law, J. High Energy Phys. 2012(10), 21 (2012).

[74] J. Erdmenger, B. Herwerth, S. Klug, R. Meyer, and K. Schalm, S-wave superconductivity in anisotropic holographic insulators, http://arxiv.org/ abs/1501.07615
[75]C.C. Homes, S.V. Dordevic, M. Strongin, D.A. Bonn, R. Liang, W.N. Hardy, S. Koymia, Y. Ando, G. Yu, X. Zhao, M. Greven, D.N. Basov, and T. Timusk, A universal scaling relation in high-temperature superconductors, Nature (London) 430(6999), 539 (2004).

[76] V.G. Kogan, Homes scaling and BCS, Phys. Rev. B 87, 220507 (2013).

[77] T. Schneider and H. Keller, Extreme type II superconductors: Universal trends, Phys. B Condens. Matter 194-196, 1789 (1994).

[78] K.-Y. Kim, K.K. Kim, and M. Park, Ward identity and Homes' law in a holographic superconductor with momentum relaxation, http://arxiv.org/ abs/1604.06205

[79] E. Kiritsis and L. Li, Holographic competition of phases and superconductivity, http://arxiv.org/ abs/1510.00020

[80] J.-W. Chen, S.-H. Dai, D. Maity, and Y.-L. Zhang, Engineering holographic superconductor phase diagrams, http://arxiv.org/abs/1603.08259

[81] J.D. Rameau, T.J. Reber, H.-B. Yang, S. Akhanjee, G.D. Gu, S. Campbell, and P.D. Johnson, Nearly perfect fluidity in a high temperature superconductor, Phys. Rev. B90, 134509 (2014).

[82]P. Kovtun, D.T. Son, and A.O. Starinets, Viscosity in strongly interacting quantum field theories from black hole physics, Phys. Rev. Lett. 94, 111601 (2005).

[83] D.T. Son and A.O. Starinets, Minkowski-space correlators in AdS/CFT correspondence: Recipe and applications, http://arxiv.org/abs/hep-th/0205051

[84] A. Rebhan and D. Steineder, Violation of the holographic viscosity bound in a strongly coupled anisotropic plasma, http://arxiv.org/abs/1110.6825

[85] K.A. Mamo, Holographic RG flow of the shear viscosity to entropy density ratio in strongly coupled anisotropic plasma, J. High Energy Phys. 2012(10), 070 (2012).

[86] R.C. Myers, T. Sierens, and W. Witczak-Krempa, A holographic model for quantum critical responses, http://arxiv.org/abs/1602.05599

[87] S. Sachdev and J. Ye, Gapless spin-fluid ground state in a random quantum Heisenberg magnet, Phys. Rev. Lett. 70, 3339 (1993).

[88] A. Georges, O. Parcollet, and S. Sachdev, Quantum fluctuations of a nearly critical Heisenberg spin glass, Phys. Rev. B 63, 4406 (2001).

[89]A. Georges, O. Parcollet, and S. Sachdev, Mean field theory of a quantum Heisenberg spin glass, Phys. Rev. Lett. 85, 840 (2000).

[90]D. Anninos, S.A. Hartnoll, L. Huijse, and V.L. Martin, Large N matrices from a nonlocal spin system, http://arxiv.org/abs/1412.1092

[91]L.F. Cugliandolo, J. Kurchan, G. Parisi, and F. Ritort, Matrix models as solvable glass models, Phys. Rev. Lett. 74, 1012C (1995). 
[92]M. Masuku, M. Mulokwe, and J.P. Rodrigues, Large $\mathrm{N}$ matrix hyperspheres and the gauge-gravity correspondence, http://arxiv.org/abs/1411.5786

[93] S. Sachdev, Strange metals and the AdS/CFT correspondence, J. Stat. Mech. 1011, P11022 (2010).

[94] J.M. Magan, Black holes as random particles: entanglement dynamics in infinite range and matrix models, http://arxiv.org/abs/1601.04663

[95]C. Bonfield, T. Wartz, J. Schirmer, and D.V. Khveshchenko [in progress].

[96] L.A. Takhtajan, The picture of low-lying excitations in the isotropic Heisenberg chain of arbitrary spins, Phys. Lett. A 87, 479 (1982).

[97]H.M. Babujian, Exact solution of the isotropic Heisenberg chain with arbitrary spins: Thermodynamics of the model, Nucl. Phys. B 215, 317B (1983).

[98] H.M. Babujian, Exact solution of the one-dimensional isotropic Heisenberg chain with arbitrary spins S, Phys. Lett. A 90, 479 (1982).

[99] A. Shnirman and Yu. Makhlin, Spin-spin correlators in Majorana representation, Phys. Rev. Lett. 91, 207204 (2003).

[100] R.S. Whitney, Yu. Makhlin, A. Shnirman, and Y. Gefen, Geometric nature of the environmentinduced Berry phase and geometric dephasing, Phys. Rev. Lett. 94, 070407 (2005).

[101] P. Schad, A. Shnirman, and Yu. Makhlin, Using Majorana spin-1/2 representation for the spin-boson model, http://arxiv.org/abs/1504.05094

[102] P. Schad, Yu. Makhlin, B.N. Narozhny, G. Schön, and A. Shnirman, Majorana representation for dissipative spin systems, Annals Phys. 361, 401 (2015).

[103] P. Benincasa and A.V. Ramallo, Holographic Kondo model in various dimensions, J. High Energy Phys. 2012(06), 133 (2012).

[104] S. Harrison, Sh. Kachru, and G. Torroba, A maximally supersymmetric Kondo model, http://arxiv. org/abs/1110.5325

[105] J. Erdmenger, C. Hoyos, A. O'Bannon, and J. Wu, A holographic model of the Kondo effect, J. High Energy Phys. 2013(12), 086 (2013), http:// arxiv.org/abs/1310.3271

[106] J. Erdmenger, M. Flory, and M.-N. Newrzella, Bending branes for DCFT in two dimensions, http://arxiv.org/abs/1410.7811

[107] J. Erdmenger, M. Flory, C. Hoyos, M.-N. Newrzella, and J.M.S. Wu, Entanglement entropy in a holographic Kondo model, http://arxiv.org/ abs/1511.03666

[108] S. Ryu and T. Takayanagi, Holographic derivation of entanglement entropy from the anti-de Sitter space/conformal field theory correspondence, Phys. Rev. Lett. 96, 181602 (2006), http:// arxiv.org/abs/hep-th/0603001

[109] T. Takayanagi, Holographic dual of a boundary conformal field theory, Phys. Rev. Lett. 107, 101602 (2011).
[110] H. Matsueda, Emergent general relativity from Fisher information metric, http://arxiv.org/ abs/1310.1831

[111] S.A. Hosseini Mansoori, B. Mirza, and M. Fazel, Hessian matrix, specific heats, Nambu brackets, and thermodynamic geometry, J. High Energy Phys. 2015(04), 115 (2015).

[112] B.-B. Wei, Z.-F. Jiang, and R.-B. Liu, Thermodynamic holography, http://arxiv.org/ abs/1411.6342

[113] P. Kumar and T. Sarkar, Geometric critical exponents in classical and quantum phase transitions, Phys. Rev. E 90, 042145 (2014).

[114] J. Molina-Vilaplana, Information geometry of entanglement renormalization for free quantum fields, J. High Energy Phys. 2015(09), 002 (2015).

[115] Y. Hashizume and H. Matsueda, Information geometry for Husimi-Temperley model, http:// arxiv.org/abs/1407.2667

[116] X.-L. Qi, Exact holographic mapping and emergent space-time geometry, http://arxiv.org/ abs/1309.6282

[117] W. Li and T. Takayanagi, Holography and entanglement in flat spacetime, Phys. Rev. Lett. 106, 141301 (2011).

[118] X. Huang and F.-L. Lin, Entanglement renormalization and integral geometry, http://arxiv.org/ abs/1507.04633

[119] M. Miyaji, T. Numasawa, N. Shiba, T. Takayanagi, and K. Watanabe, Gravity dual of quantum information metric, http://arxiv.org/abs/1507.07555

[120] D. Sels and M. Wouters, Quantum statistical gravity: time dilation due to local information in many-body quantum systems, http://arxiv.org/ abs/1602.05707

[121] B. Yang, Z. Papic, E.H. Rezayi, R.N. Bhatt, and F.D.M. Haldane, Band mass anisotropy and the intrinsic metric of fractional quantum Hall systems, Phys. Rev. B 85, 165318 (2012).

[122] A. Ghazaryan and T. Chakraborty, Emergent geometry fluctuation in quantum confined electron systems, http://arxiv.org/abs/1403.6485

[123] R.R. Biswas, Semiclassical theory of viscosity in quantum Hall states, http://arxiv.org/ abs/1311.7149

[124] T. Neupert, C. Chamon, and C. Mudry, How to measure the quantum geometry of Bloch bands, Phys. Rev. B 87, 245103 (2013), http://arxiv.org/ abs/1303.4643

[125] S. Matsuura and S. Ryu, Momentum space metric, non-local operator, and topological insulators, Phys. Rev. B 82, 245113 (2010).

[126] D. Bauer, T.S. Jackson, and R. Roy, Quantum geometry and stability of the fractional quantum Hall effect in the Hofstadter model, http://arxiv. org/abs/1504.07185

[127] J.M. Hickey, S. Genway, and J.P. Garrahan, Dynamical phase transitions, time-integrated 
observables and geometry of states, Phys. Rev. B 89, 054301 (2014).

[128] M. Kolodrubetz, V. Gritsev, and A. Polkovnikov, Classifying and measuring the geometry of the quantum ground state manifold, Phys. Rev. B 88, 064304 (2013).

[129] A. Mollabashi, M. Nozaki, S. Ryu, and T. Takayanagi, Holographic geometry of cMERA for quantum quenches and finite temperature, J. High Energy Phys. 2014(03), 098 (2014).

[130] D.V. Khveshchenko, Taking a critical look at holographic critical matter, Lith. J. Phys. 56, 208 (2015).

[131] J.M. Harris, Y.F. Yan, P. Matl, N.P. Ong, P.W. Anderson, T. Kimura, and K. Kitazawa, Violation of Kohler's rule in the normal-state magnetoresistance of $\mathrm{YBaCu}_{3} \mathrm{O}_{7-\delta}$ and $\mathrm{La}_{2} \mathrm{Sr}_{\mathrm{x}} \mathrm{CuO}_{4}$, Phys. Rev. Lett. 75, 1391 (1995).

[132] P.W. Anderson, Hall effect in the two-dimensional Luttinger liquid, Phys. Rev. Lett. 67, 2092 (1991).

[133] P. Coleman, A.J. Schofield, and A.M. Tsvelik, Phenomenological transport equation for the $\mathrm{cu}-$ prate metals, Phys. Rev. Lett. 76, 1324 (1996).

[134] C.M. Varma, P.B. Littlewood, S. SchmittRink, E. Abrahams, and A.E. Ruckenstein, Phenomenology of the normal state of $\mathrm{Cu}-\mathrm{O}$ hightemperature superconductors, Phys. Rev. Lett. 64, 497 (1990).

[135] J.W. Loram, K.A. Mirza, J.M. Wade, J.R. Cooper, and W.Y. Liang, The electronic specific heat of cuprate superconductors, Physica C 235-240, 134 (1994), http://dx.doi.org/10.1016/09214534(94)91331-5

[136] A. Karch, Conductivities for hyperscaling violating geometries, J. High Energy Phys. 2014(06), 140 (2014).

[137] S.A. Hartnoll and A. Karch, Scaling theory of the cuprate strange metals, Phys. Rev. B 91, 155126 (2015).

[138] D.V. Khveshchenko, Viable phenomenologies of the normal state of cuprates, Europhys. Lett. 111, 17005 (2015).

[139] A.V. Chubukov, D.L. Maslov, and V.I. Yudson, Optical conductivity of a two-dimensional metal at the onset of spin-density-wave order, http:/ arxiv.org/abs/1401.1461

[140] D.L. Maslov, V.I. Yudson, and A.V. Chubukov, Resistivity of a non-Galilean-invariant Fermi liquid near Pomeranchuk quantum criticality, Phys. Rev. Lett. 106, 106403 (2011).

[141] H.K. Pal, V.I. Yudson, and D.L. Maslov, Resistivity of non-Galilean-invariant Fermi- and non-Fermi liquids, Lith. J. Phys. 52, 142 (2012).

[142] M.-Y. Choi and J.S. Kim, Thermopower of high-T cuprates, Phys. Rev. B 59, 000192 (1999).

[143] Y. Zhang, N.P. Ong, P.W. Anderson, D.A. Bonn, R. Liang, and W.N. Hardy, Determining the Wiedemann-Franz ratio from the thermal Hall conductivity: Application to $\mathrm{Cu}$ and $\mathrm{YBa}_{2} \mathrm{Cu}_{3} \mathrm{O}_{6.95}$, Phys. Rev. Lett. 84, 2219 (2000).

[144] Y. Wang, L. Li, and N.P. Ong, The Nernst effect in high-T superconductors, Phys. Rev. B 73, 024510 (2006).

[145] M. Matusiak and Th. Wolf, Lorenz number in the optimally doped and underdoped superconductor $\mathrm{EuBa}_{2} \mathrm{Cu}_{3} \mathrm{O}_{\mathrm{y}}$, Phys. Rev. B 72, 054508 (2005).

[146] M. Matusiak, J. Hori, and T. Suzuki, The HallLorenz number in the $\mathrm{La}_{1.855} \mathrm{Sr}_{0.145} \mathrm{CuO}_{4}$ single crystal, Solid State Comm. 139, 376 (2006).

[147] M. Matusiak, K. Rogacki, and B.W. Veal, Enhancement of the Hall-Lorenz number in optimally doped $\mathrm{YBa}_{2} \mathrm{Cu}_{3} \mathrm{O}_{7-\mathrm{d}}$, Europhys. Lett. 88, 47005 (2009).

[148] B. Goutéraux, Universal scaling properties of extremal cohesive holographic phases, J. High Energy Phys. 2014(01), 080 (2014).

[149] B. Goutéraux, Charge transport in holography with momentum dissipation, J. High Energy Phys. 2014(04), 181 (2014).

[150] N. Barisic, M.K. Chan, M.J. Veit, C.J. Dorow, Y. Ge, Y. Tang, W. Tabis, G. Yu, X. Zhao, and M. Greven, Hidden Fermi-liquid behaviour throughout the phase diagram of the cuprates, http://arxiv.org/abs/1507.07885

[151] G. Grissonnanche, F. Laliberte, S. DufourBeausejour, M. Matusiak, S. Badoux, F.F. Tafti, B. Michon, A. Riopel, O. Cyr-Choiniere, J.C. Baglo, B.J. Ramshaw, R. Liang, D.A. Bonn, W.N. Hardy, S. Kramer, D. LeBoeuf, D. Graf, N. DoironLeyraud, and L. Taillefer, Wiedemann-Franz law in the underdoped cuprate superconductor $\mathrm{YBa}_{2} \mathrm{Cu}_{3} \mathrm{O}_{y^{\prime}}$ http://arxiv.org/abs/1503.07572

[152] G. Grissonnanche, F. Laliberte, S. DufourBeausejour, A. Riopel, S. Badoux, M. CaouetteMansour, M. Matusiak, A. Juneau-Fecteau, P. Bourgeois-Hope, O. Cyr-Choiniere, J.C. Baglo, B.J. Ramshaw, R. Liang, D.A. Bonn, W.N. Hardy, S. Kramer, D. LeBoeuf, D. Graf, N. DoironLeyraud, and L. Taillefer, Onset field for Fermisurface reconstruction in the cuprate superconductor YBCO, http://arxiv.org/abs/1508.05486

[153] A. Karch, Multiband models for field theories with anomalous current dimension, http://arxiv. org/abs/1504.02478

[154] A. Karch, K. Limtragool, and P.W. Phillips, Unparticles and anomalous dimensions in the cuprates, http://arxiv.org/abs/1511.02868

[155] K. Limtragool and P.W. Phillips, Anomalous dimension of the electrical current in the normal state of the cuprates from the fractional AharonovBohm effect, http://arxiv.org/abs/1601.02340

[156] A. Amoretti, M. Baggioli, N. Magnoli, and D. Musso, Chasing the cuprates with dilatonic dyons, http://arxiv.org/abs/1603.03029 
[157] E. Abrahams, J. Schmalian, and P. Wölfle, Strong coupling theory of heavy fermion criticality, Phys. Rev. B 90, 045105 (2014).

[158] M. Vojta, R. Bulla, and P. Wölfle, Critical quasiparticles in single-impurity and Kondo lattice models, Eur. Phys. J. Special Topics 224, 1127 (2015).

[159] P. Wölfle and E. Abrahams, Spin-flip scattering of critical quasiparticles and the phase diagram of $\mathrm{YbRh}_{2} \mathrm{Si}_{2}$, http://arxiv.org/abs/1506.08476, http:// dx.doi.org/10.1103/PhysRevB.92.155111

[160] T.R. Kirkpatrick and D. Belitz, Pre-asymptotic critical behaviour and effective exponents in disordered metallic quantum ferromagnets, Phys. Rev. Lett. 113, 127203 (2014).

[161] T.R. Kirkpatrick and D. Belitz, Exponent relations at quantum phase transitions, with applications to metallic quantum ferromagnets, Phys. Rev. B 91, 214407 (2015).

[162] T.R. Kirkpatrick and D. Belitz, Quantum correlations in metals that grow in time and space, Phys. Rev. B 93, 125130 (2016), http://arxiv.org/ abs/1508.01830

[163] S.A. Maier and P. Strack, Universality in antiferromagnetic strange metals, http://arxiv.org/ abs/1510.01331

[164] P. Kovtun and A. Ritz, Universal conductivity and central charges, Phys. Rev. D 78, 066009 (2008).

[165] P.M. Hogan and A.G. Green, Universal nonlinear conductivity near to an itinerant-electron ferromagnetic quantum critical point, Phys. Rev. B 78, 195104 (2008).

[166] N. Iqbal and H. Liu, Universality of the hydrodynamic limit in AdS/CFT and the membrane paradigm, Phys. Rev. D 79, 025023 (2009), http:// arxiv.org/abs/0809.3808

[167] S. Jain, Universal thermal and electrical conductivity from holography, J. High Energy Phys. 2010(11), 092 (2010).

[168] S. Jain, Universal properties of thermal and electrical conductivity of gauge theory plasma from holography, J. High Energy Phys. 2010(06), 023 (2010).

[169] S. Jain, Holographic electrical and thermal conductivity in strongly coupled gauge theory with multiple chemical potentials, J. High Energy Phys. 2010(03), 101 (2010).

[170] T. Faulkner, N. Iqbal, H. Liu, J. McGreevy, and D. Vegh, Charge transport by holographic Fermi surfaces, Phys. Rev. D 8, 045016 (2013).

[171] S.A. Hartnoll and D.M. Hofman, Locally critical umklapp scattering and holography, Phys. Rev. Lett. 108, 241601 (2012).

[172] A. Donos and S.A. Hartnoll, Metal-insulator transition in holography, Nat. Phys. 9, 649 (2013).

[173] A. Donos and S.A. Hartnoll, Universal linear in temperature resistivity from black hole superradiance, Phys. Rev. D 86, 124046 (2012).
[174] K. Balasubramanian and C.P. Herzog, Losing forward momentum holographically, http://arxiv. org/abs/1312.4953

[175] S.A. Hartnoll and J.E. Santos, Disordered horizons: Holography of randomly disordered fixed points, http://arxiv.org/abs/1402.0872

[176] J.-R. Sun, S.-Y. Wu, and H.-Q. Zhang, Mimic the optical conductivity in disordered solids via gauge/gravity duality, Phys. Lett. B 729, 177 (2014).

[177] A. Donos and J.P. Gauntlett, Novel metals and insulators from holography, I. High Energy Phys. 2014(06), 007 (2014), http://arxiv.org/ abs/1401.5077

[178] M. Blake and D. Tong, Universal resistivity from holographic massive gravity, Phys. Rev. D 88, 106004 (2013).

[179] T. Andrade and B. Withers, A simple holographic model of momentum relaxation, http:// arxiv.org/abs/1311.5157

[180] M. Blake, D. Tong, and D. Vegh, Holographic lattices give the graviton a mass, Phys. Rev. Lett. 112, 071602 (2014).

[181] M. Blake and A. Donos, Quantum critical transport and the Hall angle, http://arxiv.org/ abs/1406.1659

[182] S.A. Hartnoll, Theory of universal incoherent metallic transport, http://arxiv.org/abs/1405.3651

[183] A. Amoretti, A. Braggio, N. Magnoli, and D. Musso, Bounds on charge and heat diffusivities in momentum dissipating holography, J. High Energy Phys. 2015(07), 102 (2015).

[184] M. Baggioli and O. Pujolas, Holographic polarons, the metal-insulator transition and massive gravity, Phys. Rev. Lett. 114, 251602 (2015).

[185] G. Gur-Ari, S.A. Hartnoll, and R. Mahajan, Transport in Chern-Simons-matter theories, http://arxiv.org/abs/1605.01122

[186] E. Banks, A. Donos, and J.P. Gauntlett, Thermoelectric DC conductivities and Stokes flows on black hole horizons, I. High Energy Phys. 2015(10), 103 (2015), http://arxiv.org/ abs/1507.00234

[187] A. Donos and J.P. Gauntlett, The thermoelectric properties of inhomogeneous holographic lattices, J. High Energy Phys. 2015(01), 035 (2015), http://arxiv.org/abs/1409.6875

[188] A. Donos and J.P. Gauntlett, Navier-Stokes equations on black hole horizons and DC thermoelectric conductivity, Phys. Rev. D 92, 121901 (2015), http://arxiv.org/abs/1506.01360

[189] A. Donos, J.P. Gauntlett, T. Griffin, and L. Melgar, DC conductivity of magnetised holographic matter, J. High Energy Phys. 2016(01), 113 (2016), http://arxiv.org/abs/1511.00713

[190] S. Bhattacharyya, V.E. Hubeny, S. Minwalla, and M. Rangamani, Nonlinear fluid dynamics from gravity, J. High Energy Phys. 2008(02), 045 (2008), http://arxiv.org/abs/0712.2456 
[191] M. Rangamani, Gravity and hydrodynamics: Lectures on the fluid-gravity correspondence, Classical Quant. Grav. 26, 224003 (2009).

[192] V.E. Hubeny, The fluid/gravity correspondence: A new perspective on the membrane paradigm, Classical Quant. Grav. 28, 114007 (2011).

[193] V.E. Hubeny, S. Minwalla, and M. Rangamani, in: Black Holes in Higher Dimensions, ed. G.T. Horowitz (Cambridge University Press, Cambridge, 2012).

[194] R.A. Davison, Momentum relaxation in holographic massive gravity, Phys. Rev. D 88, 086003 (2013).

[195] R.A. Davison, B. Goutéraux, and S.A. Hartnoll, Incoherent transport in clean quantum critical metals, http://arxiv.org/abs/1507.07137

[196] M. Blake, Momentum relaxation from the fluid/gravity correspondence, J. High Energy Phys. 2015(09), 010 (2015).

[197] M. Blake, Universal charge diffusion and the butterfly effect,http://arxiv.org/abs/1603.08510

[198] M. Blake, Universal diffusion in incoherent black holes, http://arxiv.org/abs/1604.01754

[199] R.A. Davison and B. Goutéraux, Dissecting holographic conductivities, J. High Energy Phys. 2015(09), 090 (2015).

[200] R.A. Davison and B. Goutéraux, Momentum dissipation and effective theories of coherent and incoherent transport, J. High Energy Phys. 2015(01), 039 (2015), http://dx.doi.org/10.1007/JHEP01(2015)039

[201] A. Lucas, S. Sachdev, and K. Schalm, Scaleinvariant hyperscaling-violating holographic theories and the resistivity of strange metals with random-field disorder, http://arxiv.org/abs/1401.7993

[202] S.A. Hartnoll, R. Mahajan, M. Punk, and S. Sachdev, Transport near the Ising-nematic quantum critical point of metals in two dimensions, http://arxiv.org/abs/1401.7012

[203] A. Eberlein, I. Mandal, and S. Sachdev, Hyperscaling violation at the Ising-nematic quantum critical point in two dimensional metals, http://arxiv.org/abs/1605.00657

[204] J. Zaanen, Superconductivity: Why the temperature is high, Nature (London) 430(6999), 512 (2004).

[205] R.A. Davison, K. Schalm, and J. Zaanen, Holographic duality and the resistivity of strange metals, Phys. Rev. B 89, 245116 (2014).

[206] D.V. Khveshchenko, Searching for non-Fermi liquids under holographic light, Phys. Rev. B 86, 115115 (2012).

[207] D.-W. Pang, Probing holographic semilocal quantum liquids with D-branes, Phys. Rev. D 88, 046002 (2013).

[208] M. Edalati and J.F. Pedraza, Aspects of current correlators in holographic theories with hyperscaling violation, Phys. Rev. D 88, 086004 (2013).
[209] P. Dey and S. Roy, Zero sound in strange metals with hyperscaling violation from holography, http://arxiv.org/abs/1307.0195

[210] S.A. Hartnoll, P.K. Kovtun, M. Mueller, and S. Sachdev, Theory of the Nernst effect near quantum phase transitions in condensed matter, and in dyonic black holes, Phys. Rev. B 76, 144502 (2007).

[211] K.-Y. Kim, K.K. Kim, Y. Seo, and S.-J. Sin, Thermoelectric conductivities at finite magnetic field and the Nernst effect, http://arxiv.org/ abs/1502.05386

[212] K. Landsteiner, Y. Liu, and Y.-W. Sun, Negative magnetoresistivity in chiral fluids and holography, http://arxiv.org/abs/1410.6399

[213] M. Blake, A. Donos, and N. Lohitsiri, Magnetothermoelectric response from holography, J. High Energy Phys. 2015(08), 124 (2015).

[214] A. Amoretti, A. Braggio, N. Maggiore, N. Magnoli, and D. Musso, Analytic DC thermo-electric conductivities in holography with massive gravitons, Phys. Rev. D 91, 025002 (2015).

[215] A. Amoretti and D. Musso, Magneto-transport from momentum dissipating holography, http:// arxiv.org/abs/1502.02631

[216] H. Bantilan, J.T. Brewer, T. Ishii, W.E. Lewis, and P. Romatschke, String theory based predictions for novel collective modes in strongly interacting Fermi gases, http://arxiv.org/abs/1605.00014

[217] S. Grozdanov, N. Kaplis, and A.O. Starinets, From strong to weak coupling in holographic models of thermalization, http://arxiv.org/ $\mathrm{abs} / 1605.02173$

[218] P. Jung and A. Rosch, Lower bounds for the conductivities of correlated quantum systems, Phys. Rev. B 75, 245104 (2007).

[219] A. Lucas and S. Sachdev, Conductivity of weakly disordered strange metals: From conformal to hyperscaling-violating regimes, Nucl. Phys. B 892, 239 (2015).

[220] A. Lucas, Conductivity of a strange metal: From holography to memory functions, J. High Energy Phys. 2015(03), 071 (2015).

[221] A. Lucas and S. Sachdev, Memory matrix theory of magnetotransport in strange metals, Phys. Rev. B 91, 195122 (2015).

[222] A. Lucas, Hydrodynamic transport in strongly coupled disordered quantum field theories, http:// arxiv.org/abs/1506.02662

[223] S. Grozdanov, A. Lucas, S. Sachdev, and K. Schalm, Absence of disorder-driven metal-insulator transitions in simple holographic models, Phys. Rev. Lett. 115, 221601 (2015), http://arxiv.org/ abs/1507.00003

[224] S. Grozdanov, A. Lucas, and K. Schalm, Incoherent thermal transport from dirty black holes, http://arxiv.org/abs/1511.05970 
[225] T.N. Ikeda, A. Lucas, and Y. Nakai, Conductivity bounds in probe brane models, http://arxiv.org/ abs/1601.07882

[226] X-H. Ge, S-J. Sin, and S-F. Wu, Lower bound of electrical conductivity from holography, http:// arxiv.org/abs/1512.01917

[227] M. Baggioli and O. Pujolas, On holographic disorder-driven metal-insulator transitions, http:// arxiv.org/abs/1601.07897

[228] B. Goutéraux, E. Kiritsis, and W.-J. Li, Effective holographic theories of momentum relaxation and violation of conductivity bound, http://arxiv.org/ abs/1602.01067

[229] M. Baggioli and O. Pujolas, On effective holographic Mott insulators, https://arxiv.org abs/1604.08915

[230] E. Abrahams, S.V. Kravchenko, and M.P. Sarachik, Metallic behaviour and related phenomena in two dimensions, Rev. Mod. Phys. 73, 251 (2001).

[231] S.V. Kravchenko and M.P. Sarachik, Metal-insulator transition in two-dimensional electron systems, Rep. Progr. Phys. 67, 1 (2004).

[232] S.V. Kravchenko and M.P. Sarachik, A metalinsulator transition in 2D: Established facts and open questions, http://arxiv.org/abs/1003.2968

[233] B. Spivak, S.V. Kravchenko, S.A. Kivelson, and X.P.A. Gao, Colloquium: Transport in strongly correlated two dimensional electron fluids, Rev. Mod. Phys. 82, 1743S (2010).

[234] K. Damle and S. Sachdev, Non-zero temperature transport near quantum critical points, Phys. Rev. B 56, 8714 (1997).

[235] S. Sachdev, Nonzero temperature transport near fractional quantum Hall critical points, Phys. Rev. B 57, 7157 (1998).

[236] K. Damle and S. Sachdev, Spin dynamics and transport in gapped one-dimensional Heisenberg antiferromagnets at nonzero temperatures, Phys. Rev. B 57, 8307 (1998).

[237] M. Mueller, L. Fritz, and S. Sachdev, Quantumcritical relativistic magnetotransport in graphene, Phys. Rev. B 78, 115406 (2008).

[238] L. Fritz, J. Schmalian, M. Mueller, and S. Sachdev, Quantum critical transport in clean graphene, Phys. Rev. B 78, 085416 (2008).

[239] L. Fritz, Quantum-critical transport at a semimetal-to-insulator transition on the honeycomb lattice, Phys. Rev. B 83, 035125 (2011).

[240] B.N. Narozhny, I.V. Gornyi, M. Titov, M. Schütt, and A.D. Mirlin, Hydrodynamics in graphene: Linear-response transport, Phys. Rev. B 91, 035414 (2015).

[241] U. Briskot, M. Schütt, I.V. Gornyi, M. Titov, B.N. Narozhny, and A.D. Mirlin, Collision-dominated nonlinear hydrodynamics in graphene, Phys. Rev. B 92, 115426 (2015).

[242] M. Schütt, P.M. Ostrovsky, M. Titov, I.V. Gornyi, B.N. Narozhny, and A.D. Mirlin, Coulomb drag in graphene near the Dirac point, Phys. Rev. Lett. 110, 026601 (2013).

[243] M. Titov, R.V. Gorbachev, B.N. Narozhny, T. Tudorovskiy, M. Schuett, P.M. Ostrovsky, I.V. Gornyi, A.D. Mirlin, M.I. Katsnelson, K.S. Novoselov, A.K. Geim, and L.A. Ponomarenko, Giant magneto-drag in graphene at charge neutrality, Phys. Rev. Lett. 111, 166601 (2013).

[244] A.B. Kashuba, Conductivity of defectless graphene, Phys. Rev. B 78, 085415 (2008).

[245] M.S. Foster and I.L. Aleiner, Slow imbalance relaxation and thermoelectric transport in graphene, Phys. Rev. B 79, 085415 (2009).

[246] H.-Y. Xie and M.S. Foster, Transport coefficients of graphene: Interplay of impurity scattering, Coulomb interaction, and optical phonons, http://arxiv.org/abs/1601.05862

[247] J.M. Link, P.P. Orth, D.E. Sheehy, and J. Schmalian, Universal collisionless transport of graphene, http://arxiv.org/abs/1511.05984

[248] F. Herbut, V. Juričić, and O. Vafek, Coulomb interaction, ripples, and the minimal conductivity of graphene, Phys. Rev. Lett. 100, 046403 (2008).

[249] E.G. Mishchenko, Minimal conductivity in graphene: Interaction corrections and ultraviolet anomaly, Europhys. Lett. 83, 17005 (2008).

[250] D.E. Sheehy and J. Schmalian, Quantum critical scaling in graphene, Phys. Rev. Lett. 99, 226803 (2007).

[251] D.E. Sheehy and J. Schmalian, Optical transparency of graphene as determined by the fine-structure constant, Phys. Rev. B 80, 193411 (2009).

[252] V. Jurčić, O. Vafek, and I.F. Herbut, Conductivity of interacting massless Dirac particles in graphene: Collisionless regime, Phys. Rev. B 82, 235402 (2010).

[253] S. Teber and A.V. Kotikov, Interaction corrections to the minimal conductivity of graphene via dimensional regularization, Europhys. Lett. 107, 57001 (2014).

[254] D.A. Bandurin, I. Torre, R. Krishna Kumar, M. Ben Shalom, A. Tomadin, A. Principi, G.H. Auton, E. Khestanova, K.S. Novoselov, I.V. Grigorieva, L.A. Ponomarenko, A.K. Geim, and M. Polini, Negative local resistance caused by viscous electron backflow in graphene, Science 351, 1055 (2016).

[255] A. Lucas, J. Crossno, K.C. Fong, P. Kim, and S. Sachdev, Transport in inhomogeneous quantum critical fluids and in the Dirac fluid in graphene, http://arxiv.org/abs/1510.01738

[256] A. Lucas, Sound waves and resonances in electron-hole plasma, http://arxiv.org/abs/1604.03955

[257] D.E. Kharzeev and H.-U. Yee, Anomalies and time reversal invariance in relativistic hydrodynamics: the second order and higher dimensional formulations, http://arxiv.org/abs/1105.6360

[258] D.T. Son and B.Z. Spivak, Chiral anomaly and classical negative magnetoresistance of Weyl metals, Phys. Rev. B 88, 104412 (2013). 
[259] B.Z. Spivak and A.V. Andreev, Magnetotransport phenomena related to the chiral anomaly in Weyl semimetals, http://arxiv.org/ abs/1510.01817

[260] Y.-W. Sun and Q. Yang, Negative magnetoresistivity in holography, http://arxiv.org/ abs/1603.02624

[261] A. Lucas, R.A. Davison, and S. Sachdev, Hydrodynamic theory of thermoelectric transport and negative magnetoresistance in Weyl semimetals, http://arxiv.org/abs/1604.08598

[262] V.P.J. Jacobs, S.J.G. Vandoren, and H.T.C. Stoof, Holographic interaction effects on transport in Dirac semimetals, Phys. Rev. B 90, 045108 (2014).

[263] U. Gursoy, V. Jacobs, E. Plauschinn, H. Stoof, and S. Vandoren, Holographic models for undoped Weyl semimetals, J. High Energy Phys. 2013(04), 127 (2013).

[264] V.P.J. Jacobs, S. Grubinskas, and H.T.C. Stoof, Towards a field-theory interpretation of bottomup holography, J. High Energy Phys. 2015(04), 033 (2015), http://arxiv.org/abs/1411.4051

[265] V.P.J. Jacobs, P. Betzios, U. Gursoy, and H.T.C. Stoof, Electromagnetic response of interacting Weyl semimetals, http://arxiv.org/ abs/1512.04883

[266] L. Levitov and G. Falkovich, Electron viscosity, current vortices and negative nonlocal resistance in graphene, http://arxiv.org/abs/1508.00836

[267] P.J.W. Moll, P. Kushwaha, N. Nandi, B. Schmidt, and A.P. Mackenzie, Evidence for hydrodynamic electron flow in $\mathrm{PdCoO}_{2}$, http://arxiv.org/ abs/1509.05691

[268] J. Sonner and A.G. Green, Hawking radiation and nonequilibrium quantum critical current noise, Phys. Rev. Lett. 109, 091601 (2012), http:// dx.doi.org/10.1103/PhysRevLett.109.091601

[269] D. Bernard and B. Doyon, Non-equilibrium steady-states in conformal field theory, Ann. Henri Poincaré 16, 113 (2015), http://dx.doi.org/10.1007 s00023-014-0314-8

[270] D. Bernard and B. Doyon, A hydrodynamic approach to non-equilibrium conformal field theories, http://arxiv.org/abs/1507.07474

[271] D. Bernard and B. Doyon, Energy flow in nonequilibrium conformal field theory, J. Phys. A 45, 362001 (2012).

[272] M.J. Bhaseen, B. Doyon, A. Lucas, and K. Schalm, Far from equilibrium energy flow in quantum critical systems, Nat. Phys. 11, 509 (2015).

[273] A. Kundu and S. Kundu, Steady-state physics, effective temperature dynamics in holography, http://arxiv.org/abs/1307.6607

[274] C. Karrasch, R. Ilan, and J.E. Moore, Nonequilibrium thermal transport and its relation to linear response, http://arxiv.org/abs/1211.2236

[275] I. Bakas, K. Skenderis, and B. Withers, Self-similar equilibration of strongly interact- ing systems from holography, http://arxiv.org/ abs/1512.09151

[276] A. Lucas, K. Schalm, B. Doyon, and M.J. Bhaseen, Shock waves, rarefaction waves and nonequilibrium steady states in quantum critical systems, http://arxiv.org/abs/1512.09037

[277] D.V. Khveshchenko, Simulating holographic correspondence in flexible graphene, Europhys. Lett. 104, 47002 (2013).

[278] A. Iorio, Using Weyl symmetry to make graphene a real lab for fundamental physics, http:// arxiv.org/abs/1207.6929

[279] A. Iorio, Graphene: QFT in curved spacetimes close to experiments, J. Phys. Conf. Ser. 442, 012056 (2013), http://arxiv.org/abs/1207.6929

[280] A. Iorio and G. Lambiase, The Hawking-Unruh phenomenon on graphene, http://arxiv.org/ abs/1108.2340

[281] M. Cvetic and G.W. Gibbons, Graphene and the Zermelo optical metric of the BTZ black hole, http://arxiv.org/abs/1202.2938

[282] P. Chen and H.C. Rosu, Note on HawkingUnruh effects in graphene, http://arxiv.org/ abs/1205.4039

[283] A. Capolupo and G. Vitiello, Probing Hawking and Unruh effects and quantum field theory in curved space by geometric invariants, http://arxiv. org/abs/1311.2892

[284] M.A. Zubkov and G.E. Volovik, Emergent gravity in graphene, http://arxiv.org/abs/1308.2249

[285] M.A.H. Vozmediano, M.I. Katsnelson, and F. Guinea, Gauge fields in graphene, Phys. Rep. 496, 109 (2010).

[286] F. de Juan, A. Cortijo, and M.A.H. Vozmediano, Dislocations and torsion in graphene and related systems, Nucl. Phys. B 828, 625 (2010).

[287] F. de Juan, A. Cortijo, M.A.H. Vozmediano, and A. Cano, Aharonov-Bohm interferences from local deformations in graphene, http://arxiv.org/ abs/1105.0599

[288] M.I. Katsnelson, F. Guinea, and M.A.H. Vozmediano, Gauge fields at the surface of topological insulators, http://arxiv.org/abs/1105.6132

[289] A.L. Kitt, V.M. Pereira, A.K. Swan, and B.B. Goldberg, Lattice-corrected strain-induced vector potentials in graphene, Phys. Rev. B 85, 115432 (2012).

[290] F. de Juan, M. Sturla, and M.A.H. Vozmediano, Space dependent Fermi velocity in strained graphene, Phys. Rev. Lett. 108, 227205 (2012).

[291] F. de Juan, J.L. Mañes, and M.A.H. Vozmediano, Gauge fields from strain in graphene, Phys. Rev. B 87, 165131 (2013).

[292] M. Ramezani Masir, D. Moldovan, and F.M. Peeters, Pseudo magnetic field in strained graphene: Revisited, http://arxiv.org/abs/1304.0629

[293] M. Oliva-Leyva and G.G. Naumis, Understanding electron behaviour in strained graphene 
as a reciprocal space distortion, http://arxiv.org/ abs/1304.6682

[294] B. Reznik, Origin of the thermal radiation in a solid-state analog of a black-hole, http://arxiv. org/abs/gr-qc/9703076

[295] W. Lu, J. Jin, H. Chen, and Z. Lin, A simple design of an artificial electromagnetic black hole, http://arxiv.org/abs/1003.5727

[296] M. Li, R.-X. Miao, and Y. Pang, Casimir energy, holographic dark energy and electromagnetic metamaterial mimicking de Sitter, http://arxiv.org/ abs/0910.3375

[297] M. Li and Y. Pang, Holographic de Sitter universe, https://arxiv.org/abs/1105.0038

[298] R.-X. Miao, R. Zheng, and M. Li, Metamaterials mimicking dynamic spacetime, D-brane and noncommutativity in string theory, http://arxiv.org/ abs/1005.5585

[299] T.G. Mackay and A. Lakhtakia, Towards a realization of Schwarzschild-(anti-)de Sitter spacetime as a particulate metamaterial, http://arxiv.org abs/1102.1708

[300] T.G. Mackay and A. Lakhtakia, Towards a metamaterial simulation of a spinning cosmic string, http://arxiv.org/abs/0911.4163

[301] T.-M. Zhao and R.-X. Miao, Huge Casimir effect at finite temperature in electromagnetic Rindler space, http://arxiv.org/abs/1110.1919

[302] D. Brill, Black holes and wormholes in 2+1 dimensions, http://arxiv.org/abs/gr-qc/9904083

[303] I.I. Smolyaninov, Y.-J. Hung, and E. Hwang, Experimental modeling of cosmological inflation with metamaterials, http://arxiv.org/abs/1111.3300

[304] I.I. Smolyaninov, E. Hwang, and E. Narimanov, Hyperbolic metamaterial interfaces: Hawking radiation from Rindler horizons and the "end of time", http://arxiv.org/abs/1107.4053
[305] I.I. Smolyaninov and E.E. Narimanov, Metric signature transitions in optical metamaterials, Phys. Rev. Lett. 105, 067402 (2010).

[306] I.I. Smolyaninov, Metamaterial "multiverse", J. Optics 13, 024004 (2011).

[307] I.I. Smolyaninov, Metamaterial model of tachyonic dark energy, http://arxiv.org/abs/1310.8155

[308] I.I. Smolyaninov, Analog of gravitational force in hyperbolic metamaterials, Phys. Rev. A 88, 033843 (2013), http://arxiv.org/abs/1307.8431

[309] I.I. Smolyaninov, Extra-dimensional metamaterials: simple models of inflation and metric signature transitions, http://arxiv.org/abs/1301.6060

[310] I.I. Smolyaninov, Modeling of causality with metamaterials, J. Optics 15, 025101 (2013).

[311] I.I. Smolyaninov, Holographic duality in nonlinear hyperbolic metamaterials, http://arxiv.org/ abs/1401.3242

[312] D.V. Khveshchenko, Contrasting string holography to its optical namesake, Europhys. Lett. 109, 61001 (2015).

[313] M. Novello, M. Visser, and G.E. Volovik (eds.), Artificial Black Holes (World Scientific, Singapore, 2002).

[314] R. Schützhold and W.G. Unruh (eds.), Quantum Analogues: From Phase Transitions to Black Holes and Cosmology, Lecture Notes in Physics, Vol. 718 (Springer, 2007), http://dx.doi.org/10.1007/3-540$70859-6$

[315] C. Barcelo, S. Liberati, and M. Visser, Analogue gravity, Living Rev. Rel. 14, 3 (2011) [updated from Living Rev. Rel. 8, 12 (2005)].

[316] M. Visser, in: Analogue Gravity Phenomenology, Lecture Notes in Physics, Vol. 870, eds. D. Faccio, F. Belgiorno, S. Cacciatori, V. Gorini, S. Liberati, and U. Moschella (Springer, 2013) pp. 31-50, http://dx.doi.org/10.1007/978-3-319-00266-8

\title{
HOLOGRAFIJOS TEORIJŲ DEMISTIFIKACIJA: KRITINĖ APŽVALGA
}

\author{
D.V. Khveshchenko
}

Šiaurès Karolinos universitetas, JAV 